\title{
Unprojection and deformations of tertiary Burniat surfaces
}

\author{
Jorge Neves And Roberto Pignatelli
}

\begin{abstract}
We construct a 4-dimensional family of surfaces of general type with $p_{g}=0$ and $K^{2}=3$ and fundamental group $\mathbb{Z} / 2 \times Q_{8}$, where $Q_{8}$ is the quaternion group. The family constructed contains the Burniat surfaces with $K^{2}=3$. Additionally, we construct the universal coverings of the surfaces in our family as complete intersections on $\left(\mathbb{P}^{1}\right)^{4}$ and we also give an action of $\mathbb{Z} / 2 \times Q_{8}$ on $\left(\mathbb{P}^{1}\right)^{4}$ lifting the natural action on the surfaces.

The strategy is the following. We consider an étale $(\mathbb{Z} / 2)^{3}$-cover $T$ of a surface with $p_{g}=0$ and $K^{2}=3$ and assume that it may be embedded in a Fano 3 -fold $V$. We construct $V$ by using the theory of parallel unprojection. Since $V$ is an Enriques-Fano 3-fold, considering its Fano cover yields the simple description of the above universal covers.
\end{abstract}

Mathematics Subject Classification (2010): 14J29 (primary).

\section{Introduction}

A Burniat surface is the minimal resolution of singularities of a bidouble cover, i.e., a finite flat Galois morphism with Galois group $(\mathbb{Z} / 2)^{2}$, of the projective plane branched along the divisors

$$
D_{1}=A_{1}+A_{2}+A_{3}, \quad D_{2}=B_{1}+B_{2}+B_{3}, \quad D_{3}=C_{1}+C_{2}+C_{3},
$$

where $A_{1}, B_{1}, C_{1}$ form a triangle with vertices $\mathbf{x}_{1}, \mathbf{x}_{2}, \mathbf{x}_{3}, A_{1}, A_{2}, A_{3}$ are lines through $\mathbf{x}_{1}, B_{1}, B_{2}, B_{3}$ are lines through $\mathbf{x}_{2}$ and $C_{1}, C_{2}, C_{3}$ are lines through $\mathbf{x}_{3}$ (see Figure 1.1). Burniat surfaces were first constructed by Burniat [13], though a substantial part of the initial study of these surfaces was done about 10 years later by Peters [29]. They have an equivalent description known as the Inoue surfaces [17], given as the quotient of a divisor in the product of three elliptic curves by a finite group. See [3] for an excellent introduction to the subject of Burniat surfaces.

This work was partially supported by CMUC and FCT (Portugal) through the European program COMPETE/FEDER and through Projects PTDC/MAT/099275/2008 and PTDC/MAT/111332/ 2009.

Received July 26, 2011; accepted in revised form March 20, 2012. 
Burniat surfaces are minimal surfaces of general type with $p_{g}=\operatorname{dim} H^{0}\left(\Omega^{2}\right)=0$ and hence with irregularity, $q=\operatorname{dim} H^{0}\left(\Omega^{1}\right)$, equal to 0 . The study of the moduli space of surfaces of general type with these invariants started in 1932 with Campedelli's celebrated construction of a surface of general type with $p_{g}=0$ and $K^{2}=c_{1}^{2}=2$, as a double cover of the projective plane branched along a curve of degree 10 with 6 singular points, not lying on a conic, all of type $[3,3]$, that is a triple point with another infinitely near triple point. Nowadays, this subject is still the object of much attention, with new results on the description of whole components of this moduli space (e.g. $[2,14,23,24,30,31])$ and on the proof of existence of new ones $(e . g .[6,7,9,20,22,26,28])$. See [8] for a survey on surfaces of general type with $p_{g}=0$.

Let $S$ be a Burniat surface. If we assume that the branch divisors $D_{1}, D_{2}, D_{3}$ in the configuration described earlier, besides satisfying the conditions stated there, are otherwise general, then $K_{S}^{2}=6$. By the general theory of bidouble covers (see [15]), imposing further, to the triple $D_{1}, D_{2}, D_{3}, m$ singular points of type $(1,1,1)$ (which are points which belong to each $D_{i}$, which are smooth for each $D_{i}$, and such that the three tangent directions are different), then $K^{2}$ drops by $m$ and the other invariants do not change. This yields 6 families (two for $m=2$ : the family of nodal type and the family of non nodal type) the dimensions of which are equal to $K_{S}^{2}-2=4-m$, respectively.

Following Bauer and Catanese [3], call a Burniat surface primary if $K_{S}^{2}=6$, secondary if $K_{S}^{2}=4,5$, tertiary if $K_{S}^{2}=3$ (see Figure 1.1) and quaternary if $K_{S}^{2}=$ 2 . From a certain point of view, what sets apart primary and secondary Burniat surfaces from tertiary and quaternary Burniat surfaces is that the former families have dimensions greater than or equal to the expected dimension $10 \chi\left(\mathcal{O}_{S}\right)-2 K_{S}^{2}$ of the corresponding moduli spaces, while the latter families have dimensions strictly less than the expected dimension of the corresponding moduli spaces. More precisely, the family of tertiary Burniat surfaces is 1-dimensional, whereas the moduli space

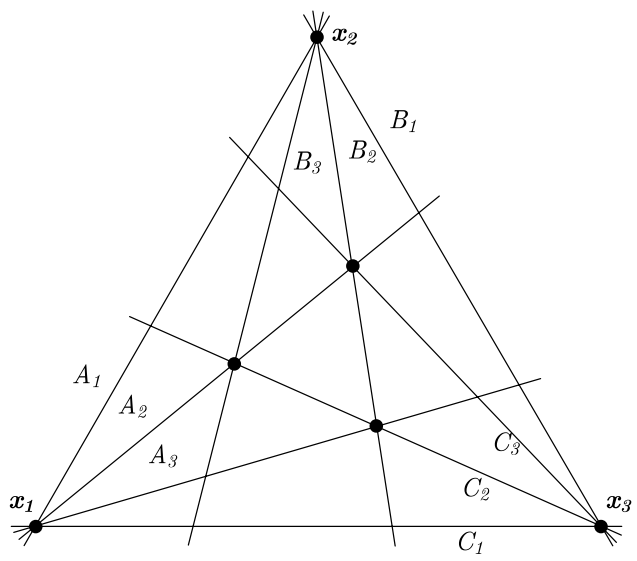

Figure 1.1. Branching divisors for tertiary Burniat surfaces. 
of surfaces of general type with $p_{g}=0$ and $K^{2}=3$ has expected dimension equal to 4 and the family quaternary Burniat surfaces is 0-dimensional, whereas the moduli space of surfaces of general type with $p_{g}=0$ and $K^{2}=2$ (the Campedelli surfaces) has expected dimension equal to 6 .

In 2001, Mendes Lopes and Pardini ( $c f$. [21]) proved that the 4-dimensional family of primary Burniat surfaces forms a normal, unirational, irreducible connected component of the moduli space of surfaces of general type with $p_{g}=0$ and $K^{2}=6$. In 2004, Kulikov ( $c f$. [18]) proved that the class of the quaternary Burniat surfaces belongs to the component of classical Campedelli surfaces, i.e., $p_{g}=0, K^{2}=2$ and torsion group $(\mathbb{Z} / 2)^{3}$, which had been completely described (cf. [25,32]). In a deep recent analysis (cf. [3-5]), Bauer and Catanese have continued the study of the components of the moduli space of surfaces of general type containing the Burniat surfaces. They gave an alternative proof of Mendes LopesPardini's result on primary Burniat surfaces. They showed that of the 3 families corresponding to secondary Burniat surfaces the one with $K^{2}=5$ and the one with $K^{2}=4$ of non nodal type form irreducible connected components. They have also described the whole connected component containing the Burniat surfaces with $K^{2}=4$ of nodal type, which turns out to have dimension 3 , one more than the expected dimension.

This article is devoted to a construction of a 4-dimensional family of minimal surfaces, $S$, of general type with $p_{g}(S)=0$ and $K_{S}^{2}=3$, containing, as a codimension 3 subfamily, the family of tertiary Burniat surfaces. We do this by constructing a 4-dimensional family of surfaces of general type $T$ with $\chi\left(\mathcal{O}_{T}\right)=8$ and $K_{T}^{2}=24$, equipped with a free $G=(\mathbb{Z} / 2)^{3}$ action. We take $S$ as the quotient $T / G$. The family of surfaces $T$ is a linear subsystem of $\left|-2 K_{V}\right|$, where $V$ is an Enriques-Fano 3 -fold in $\mathbb{P}\left(1^{7}, 2^{8}\right)$ obtained from a complete intersection Fano 3fold in $\mathbb{P}^{6}$ on which there exists an action of $G$ inducing the action of this group on $T$. In this respect, we can see $V$ as a key variety for this construction; just as weighted projective space acts as key variety in most elementary constructions. This idea is reminiscent of the construction of a numerical Campedelli surface with torsion group $\mathbb{Z} / 6$ of [26]. Lifting the action of $G$ to the Fano double cover of $V$ we obtain the simple description of our family described in the next theorem, which synthesizes Theorem 2.10, Theorem 3.5, Theorem 3.6 and Theorem 4.5 of this work.

Theorem 1.1. Consider $\mathbb{P}^{1} \times \mathbb{P}^{1} \times \mathbb{P}^{1} \times \mathbb{P}^{1}$, with coordinates $\left(t_{00}, t_{01}\right)$, $\left(t_{10}, t_{11}\right)$, $\left(t_{20}, t_{21}\right),\left(t_{30}, t_{31}\right)$ and the group $\tilde{G}<\operatorname{Aut}\left(\mathbb{P}^{1} \times \mathbb{P}^{1} \times \mathbb{P}^{1} \times \mathbb{P}^{1}\right)$ generated by the 3 automorphisms in the following table, where $\epsilon$ is a chosen square root of -1 :

\begin{tabular}{rrrrrrrrr}
\hline & $t_{00}$ & $t_{01}$ & $t_{10}$ & $t_{11}$ & $t_{20}$ & $t_{21}$ & $t_{30}$ & $t_{31}$ \\
\hline$\tilde{\alpha}_{1} \tilde{\beta}_{2}$ & $-\epsilon t_{10}$ & $t_{11}$ & $t_{00}$ & $\epsilon t_{01}$ & $t_{31}$ & $-\epsilon t_{30}$ & $t_{21}$ & $\epsilon t_{20}$ \\
\hline$\tilde{\alpha}_{2} \tilde{\beta}_{3}$ & $-\epsilon t_{20}$ & $t_{21}$ & $t_{31}$ & $\epsilon t_{30}$ & $t_{00}$ & $\epsilon t_{01}$ & $t_{11}$ & $-\epsilon t_{10}$ \\
\hline$\tilde{\alpha}_{3} \tilde{\beta}_{1}$ & $-\epsilon t_{30}$ & $t_{31}$ & $t_{21}$ & $-\epsilon t_{20}$ & $t_{11}$ & $\epsilon t_{10}$ & $t_{00}$ & $\epsilon t_{01}$ \\
\hline
\end{tabular}


Then $\tilde{G} \cong \mathbb{Z} / 2 \times Q_{8}$, where $Q_{8}$ denotes the standard quaternion group. Consider also the $\tilde{G}$-invariant hypersurface of multi-degree $(1,1,1,1)$ given by

$$
Z_{1}:=\left(t_{01} t_{10} t_{20} t_{30}+t_{00} t_{11} t_{21} t_{31}=0\right)
$$

and the $\tilde{G}$-invariant surfaces $\tilde{T}$ cut out on $Z_{1}$ by the multi-degree $(2,2,2,2)$ hypersurfaces given by

$$
Z_{2}=\sum_{i=0}^{3} v_{i}\left(t_{i 0}^{2} \prod_{j \neq i} t_{j 1}^{2}+t_{i 1}^{2} \prod_{j \neq i} t_{j 0}^{2}\right)-\underset{a+b+c+d \text { even }}{2 v_{4}} \sum_{a}(-1)^{\frac{b+c+d-a}{2}} t_{0 a}^{2} t_{1 b}^{2} t_{2 c}^{2} t_{3 d}^{2}=0
$$

for $v_{0}, v_{1}, v_{2}, v_{3}, v_{4} \in \mathbb{C}$. Then, if the $v_{i}$ are general, $\tilde{G}$ acts freely on $\tilde{T}$ and the quotient $S=\tilde{T} / \tilde{G}$ is the canonical model of a surface of general type with $p_{g}=0$, $K^{2}=3$ and $\pi_{1}(S) \cong \mathbb{Z} / 2 \times Q_{8}$. The family obtained in this way describes a 4-dimensional locus in the moduli space of the surfaces of general type, containing the tertiary Burniat surfaces, for which $-v_{0}=v_{1}=v_{2}=v_{3}$.

Note that the fundamental group of tertiary Burniat surfaces has already been computed in [3], which fixes a mistake in a previous computation in [29]. The study of surfaces of general type with $p_{g}=0, K^{2}=3$ and fundamental group of order 16 is of special interest as, according to a conjecture of M. Reid, this number should be the maximum order of their (algebraic) fundamental groups.

Our construction gives a 4-dimensional stratum of the moduli space of the surfaces of general type containing the tertiary Burniat surfaces. In [5], Bauer and Catanese prove that the irreducible component of the moduli space of surfaces of general type containing the tertiary Burniat surfaces has dimension 4, and they construct a proper open set of it; it follows that also our family forms an open set of the same component. We expect that our family is not a proper subset, covering the full irreducible component. It is also reasonable to guess that this irreducible component is a full connected component of the moduli space.

We now explain the motivation for our construction. Let $T$ be a minimal regular surface of general type with $\chi\left(\mathcal{O}_{T}\right)=8$ and $K_{T}^{2}=24$. Assume that $T \in\left|-2 K_{V}\right|$, where $V$ is a $\mathbb{Q}$-Fano 3-fold with $n$ singular points of type $\frac{1}{2}(1,1,1)$. Then $h^{0}\left(-K_{V}\right)=p_{g}=7,-K_{V}^{3}=K_{T}^{2} / 2=12$ and by the orbifold RiemannRoch formulas $(c f$. $[1,12]), 4 p_{g}=K_{T}^{2}+12-n$, i.e., $n=8$. This leads to a candidate 3-fold $V$ anticanonically embedded in $\mathbb{P}\left(1^{7}, 2^{8}\right)$ that, by the Graded Ring Database [11], projects to a complete intersection $W_{2,2,2} \subset \mathbb{P}^{6}$. On the other hand, suppose that $T$ is equipped with a free $G=(\mathbb{Z} / 2)^{3}$ action. By the Lefschetz Holomorphic Fixed Point Formula we know the character of the representation of $G$ on $H^{0}\left(n K_{T}\right)$. Throughout the paper $a, b, c, d$ vary in $\mathbb{Z} / 2=\{0,1\}$, and we will use the notation $0^{\prime}=1$ and $1^{\prime}=0$. Writing $\chi_{a b c}$, for the irreducible representations of $G$, we get:

$$
\begin{gathered}
H^{0}\left(K_{T}\right)=\bigoplus_{(a, b, c) \in G \backslash\{(0,0,0)\}} \chi_{a b c}, \quad H^{0}\left(2 K_{T}\right)=\bigoplus_{(a, b, c) \in G} \chi_{a b c}^{\oplus 4}, \\
S^{2} H^{0}\left(K_{T}\right)=\chi_{000}^{\oplus 7} \oplus \chi_{100}^{\oplus 3} \oplus \chi_{010}^{\oplus 3} \oplus \chi_{001}^{\oplus 3} \oplus \chi_{110}^{\oplus 3} \oplus \chi_{101}^{\oplus 3} \oplus \chi_{011}^{\oplus 3} \oplus \chi_{111}^{\oplus 3} .
\end{gathered}
$$


We deduce that the canonical ring of $T$,

$$
R\left(T, K_{T}\right)=\bigoplus_{n \in \mathbb{N}} H^{0}\left(n K_{T}\right)
$$

on which $G$ acts, has 3 invariant quadric relations and needs 7 new generators in degree 2, one for each of the nontrivial rank 1 representations $G$. This agrees with the properties of $V$. The anticanonical ring $R\left(V,-K_{V}\right)$ has 8 generators of degree 2 and 3 quadric relations between the degree 1 generators, coming from the defining equations of $W_{2,2,2} \subset \mathbb{P}^{6}$. Note that $R\left(T, K_{T}\right)$ can be obtained from $R\left(V,-K_{V}\right)$ by taking a quotient by a degree 2 regular element.

As in [26], the first goal is to construct $V$ from $W_{2,2,2} \subset \mathbb{P}^{6}$ using parallel unprojection, which is to say, unproject all at once 8 divisors in $W$ satisfying sufficiently general conditions.

The second goal is to set up an action of $G \cong(\mathbb{Z} / 2)^{3}$ on $\mathbb{P}\left(1^{8}, 2^{8}\right)$ that leaves $Y, V$ and $T$ invariant and is fixed point free on $T$. With this in mind we establish a $(\mathbb{Z} / 2)^{6}$ action on $\mathbb{P}\left(1^{8}, 2^{8}\right)$ which leaves $Y$ and $V$ invariant and for which there exists a subgroup $H \subset(\mathbb{Z} / 2)^{6}$ isomorphic to $(\mathbb{Z} / 2)^{5}$ which leaves $T$ invariant. We then show that $H$ has a subgroup $G \cong(\mathbb{Z} / 2)^{3}$ which acts fixed point freely on $T$. The upshot is that the quotient group $H / G \cong(\mathbb{Z} / 2)^{2}$ acts on $S:=T / G$ and the quotient map coincides with the bicanonical map of $S$. (Cf. Proposition 4.1.)

The paper is divided up as follows. In Section 2 we describe the construction of $Y \subset \mathbb{P}\left(1^{8}, 2^{8}\right)$ via parallel unprojection of a 4-fold complete intersection of 3 quadrics $X \subset \mathbb{P}^{7}$ using the format introduced in [27]. We obtain a $\mathbb{Q}$-Fano 3-fold $V \subset Y$ by taking a hypersurface section of degree 1 of $V$ and the surface $T \subset V$ by taking a hypersurface section of degree 2 of $V$. The bulk of this section is concerned with the study of the geometry of $V$ (with emphasis on its singularities) and setting up of the group action described above. In Section 3, we show that $Y$ is the quotient of $\mathbb{P}^{1} \times \mathbb{P}^{1} \times \mathbb{P}^{1} \times \mathbb{P}^{1}$ by an involution and we lift the action of $G$ to an action of $\tilde{G}=$ $\mathbb{Z} / 2 \times Q_{8}$ on $\mathbb{P}^{1} \times \mathbb{P}^{1} \times \mathbb{P}^{1} \times \mathbb{P}^{1}$. We obtain a description of our surfaces as quotient by a fixed point free action of $\tilde{G}$ on a complete intersection in $\mathbb{P}^{1} \times \mathbb{P}^{1} \times \mathbb{P}^{1} \times \mathbb{P}^{1}$, which enables the computation of their fundamental group. Finally we show that the family constructed is unirational and has 4 moduli. In Section 4 we carry out a detailed study of the bicanonical map of $S=T / G$. We show that the bicanonical map is a bidouble cover of a singular cubic surface $S_{3} \subset \mathbb{P}^{3}$ and compute the branch loci of this map. Via a birational map $S_{3}-\rightarrow \mathbb{P}^{2}$ we reinterpret this bidouble cover as a bidouble cover of $\mathbb{P}^{2}$ and use it to show that the family of surfaces constructed contains the family of tertiary Burniat surfaces.

ACKNOWLEDGEMENTS. The authors are grateful to CIRM-Trento for supporting the visit of the first author to Trento in April of 2010. We are indebted with M. Mella for pointing out the theory of Enriques-Fano 3-folds, which inspired the construction in Section 3.

The second author would like to thank I. Bauer and F. Catanese for some very interesting discussions about their work on Burniat surfaces. 


\section{The Construction of $S$}

Consider $\mathbb{P}^{7}$ with homogeneous coordinates $x_{00}, x_{01}, x_{10}, x_{11}, x_{20}, x_{21}, x_{30}, x_{31}$ and let $X \subset \mathbb{P}^{7}$ be the 4-fold complete intersection of 3 quadrics given by:

$$
x_{00} x_{01}=x_{10} x_{11}=x_{20} x_{21}=x_{30} x_{31} .
$$

Notice that $X$ contains the 16 linear spaces given by:

$$
H_{a b c d}=\left(x_{0 a}=x_{1 b}=x_{2 c}=x_{3 d}=0\right), \quad a, b, c, d \in\{0,1\}
$$

all of which have codimension 1 in $X$. These 16 linear spaces can be thought of as the vertices of the 4-cube, by identifying their equations (2.2) with the vertex $(a, b, c, d)$. An edge between two vertices means that the intersection of the corresponding linear spaces has dimension $\geq 2$ or, equivalently, that the union of the sets of equations of the linear spaces does not contain a regular sequence of length 6 .

Since the homogeneous coordinate rings of $X$ and of each linear space are Gorenstein graded rings, we can use Kustin-Miller parallel unprojection on a subset of the set of linear spaces in (2.2). Indeed the format of the equations of $X$ was studied in [27, Section 3], where a sufficient condition for the existence of the parallel unprojection was given. In our case, a subset of linear spaces can be unprojected if the defining equations of any two linear subspaces in it contain a regular sequence of length 6 . Since the 4-cube is a bipartite graph, there are 2 maximal subsets with this property. These subsets yield isomorphic constructions, thus we shall fix one. Let $\mathcal{L}$ denote the subset of $\{0,1\}^{4}$ consisting of the 4 -tuples with even sum and consider the corresponding subset of linear spaces: $\left\{H_{a b c d} \mid(a, b, c, d) \in \mathcal{L}\right\}$. Recall that throughout the paper we shall be using the following shorthand notation: $0^{\prime}=1$ and $1^{\prime}=0$.

Remark 2.1. Notice that $H_{a b c d} \cap H_{a^{\prime} b^{\prime} c^{\prime} d^{\prime}}=\emptyset$. Any other pair of distinct elements in $\left\{H_{a b c d} \mid(a, b, c, d) \in \mathcal{L}\right\}$ intersect along a line. These 24 lines form the singular locus of $X$.

According to [27, Lemma 3.2] we can perform the parallel unprojection of these 8 linear spaces in $X$, to obtain a projectively Gorenstein subscheme of a weighted projective space, $Y \subset \mathbb{P}\left(1^{8}, 2^{8}\right)$, as follows.

Definition 2.2. Consider, for each $(a, b, c, d) \in \mathcal{L}$ the rational section of $\mathcal{O}_{X}(2)$

$$
\varphi_{a b c d}:=\frac{x_{1 b^{\prime}} x_{2 c^{\prime}} x_{3 d^{\prime}}}{x_{0 a}}=\frac{x_{0 a^{\prime}} x_{2 c^{\prime}} x_{3 d^{\prime}}}{x_{1 b}}=\frac{x_{0 a^{\prime}} x_{1 b^{\prime}} x_{3 d^{\prime}}}{x_{2 c}}=\frac{x_{0 a^{\prime}} x_{1 b^{\prime}} x_{2 c^{\prime}}}{x_{3 d}},
$$

where the equalities follow from (2.1). The divisor of the poles of $\varphi_{a b c d}$ is $H_{a b c d}$.

We denote by $\varphi: X \rightarrow-\rightarrow \mathbb{P}\left(1^{8}, 2^{8}\right)$ the unprojection map, i.e., the rational map

$$
\varphi\left(x_{00}, x_{01}, \ldots, x_{31}\right)=\left(x_{00}, x_{01}, \ldots, x_{31}, \varphi_{0000}\left(x_{i a}\right), \ldots, \varphi_{1111}\left(x_{i a}\right)\right) .
$$

We define $Y:=\varphi(X)$. 
Notation 2.3. We denote accordingly the weight- 2 variables of the ambient weighted projective space by $y_{a b c d}$, where $y_{a b c d}$ is the variable corresponding to $\varphi_{a b c d}\left(x_{i a}\right)$ in the definition of $\varphi$. Let $\pi: \mathbb{P}\left(1^{8}, 2^{8}\right) \rightarrow \mathbb{P}^{7}$ denote the projection map, i.e., the rational map obtained by forgetting the degree- 2 variables.

The ideal $J$ of the subvariety $Y \subset \mathbb{P}\left(1^{8}, 2^{8}\right)$ is generated by the following homogeneous polynomials: the original 3 quadrics - given by the difference of two terms in $(2.1)-32$ cubics, given by

$$
\begin{aligned}
& y_{a b c d} x_{0 a}-x_{1 b^{\prime}} x_{2 c^{\prime}} x_{3 d^{\prime}}, \quad y_{a b c d} x_{1 b}-x_{0 a^{\prime}} x_{2 c^{\prime}} x_{3 d^{\prime}}, \\
& y_{a b c d} x_{2 c}-x_{0 a^{\prime}} x_{1 b^{\prime}} x_{3 d^{\prime}}, \quad y_{a b c d} x_{3 d}-x_{0 a^{\prime}} x_{1 b^{\prime}} x_{2 c^{\prime}},
\end{aligned}
$$

for every $(a, b, c, d) \in \mathcal{L}$; and 28 quartics, given by

$$
y_{a_{0} a_{1} a_{2} a_{3}} y_{b_{0} b_{1} b_{2} b_{3}}-\frac{x_{0 a_{0}^{\prime}} x_{1 a_{1}^{\prime}} x_{2 a_{2}^{\prime}} x_{3 a_{3}^{\prime}}}{x_{i a_{i}^{\prime}} x_{i a_{i}}} \cdot \frac{x_{0 b_{0}^{\prime}} x_{1 b_{1}^{\prime}} x_{2 b_{2}^{\prime}} x_{3 b_{3}^{\prime}}}{x_{j b_{j}^{\prime}} x_{j b_{j}}}
$$

for every distinct $\left(a_{0}, a_{1}, a_{2}, a_{3}\right),\left(b_{0}, b_{1}, b_{2}, b_{3}\right) \in \mathcal{L}$, where, given $\left(a_{0}, a_{1}, a_{2}, a_{3}\right)$, $\left(b_{0}, b_{1}, b_{2}, b_{3}\right)$ in $\mathcal{L}, i$ and $j$ are such that $a_{i} \neq b_{i}$ and $a_{j} \neq b_{j}$, so that the fractional expression of (2.5) is always a polynomial.

Remark 2.4. The unprojection map $\varphi: X \rightarrow Y$ is a birational map between $X$ and $Y$, with inverse $\pi_{\mid Y}: Y \rightarrow X$. Indeed, $\varphi$ induces an isomorphism

$$
X \backslash\left(\bigcup_{a b c d \in \mathcal{L}} H_{a b c d}\right) \rightarrow Y \backslash\left(\bigcup_{a b c d \in \mathcal{L}} \mathcal{H}_{a b c d}\right)
$$

where $\mathcal{H}_{a b c d}$ is the subscheme of $Y$ given by $x_{0 a}=x_{1 b}=x_{2 c}=x_{3 d}=0$.

Notation 2.5. Firstly we make notation for the coordinate points of $\mathbb{P}^{7}$ and $\mathbb{P}\left(1^{8}, 2^{8}\right)$. Given $0 \leq i \leq 3$ and $a \in\{0,1\}$ we denote by $\mathbf{x}_{i a}$ the point of $\mathbb{P}^{7}$, or of $\mathbb{P}\left(1^{8}, 2^{8}\right)$, depending on the context, having all but the coordinate $x_{i a}$ equal to zero. Similarly, given $(a, b, c, d) \in \mathcal{L}$, we denote by $\mathbf{y}_{a b c d} \in \mathbb{P}\left(1^{8}, 2^{8}\right)$ the point defined in an analogous way. Note that the 8 points $\mathbf{y}_{a b c d}$ are the intersection of $Y$ with the singular locus of the ambient space, and also the centers of the projection $\pi_{\mid Y}$. Secondly we establish notation for a distinguish set of surfaces in $\mathbb{P}\left(1^{8}, 2^{8}\right)$. There are 24 quartic polynomials in (2.5) involving the product of 2 squares. Such is the case with $y_{0011} y_{0000}-x_{01}^{2} x_{11}^{2}$. This polynomial defines a subscheme, $\mathcal{S}_{11}^{01}$, of dimension 2 of the 3 -dimensional projective space $\mathbb{P}\left(1^{2}, 2^{2}\right)$ with variables $x_{01}, x_{11}$, $y_{0011}, y_{0000}$ that we can regard as a subscheme $\mathcal{S}_{11}^{01} \subset \mathbb{P}\left(1^{8}, 2^{8}\right)$, by setting all but the coordinates $x_{01}, x_{11}, y_{0011}, y_{0000}$ equal to 0 . Similarly, given $0 \leq i<j \leq 3$ and $a, b \in\{0,1\}$ we denote by $\mathcal{S}_{a b}^{i j}$ the subscheme of $\mathbb{P}\left(1^{2}, 2^{2}\right) \subset \mathbb{P}\left(1^{8}, 2^{8}\right)$ defined by the quartic polynomial of (2.5) involving $x_{i a}^{2} x_{j b}^{2}$. These are 24 surfaces contained in $Y$. 
Lemma 2.6. Set-theoretically, $\mathcal{H}_{a^{\prime} b^{\prime} c^{\prime} d^{\prime}}=\left\{\mathbf{y}_{a b c d}\right\} \cup \mathcal{S}_{a b}^{01} \cup \mathcal{S}_{a c}^{02} \cup \mathcal{S}_{a d}^{03} \cup \mathcal{S}_{b c}^{12} \cup \mathcal{S}_{b d}^{13} \cup$ $\mathcal{S}_{c d}^{23}$. In particular $\mathcal{H}_{a b c d}$ is 2-dimensional, for all $(a, b, c, d) \in \mathcal{L}$.

Proof. We prove the lemma for $(a, b, c, d)=(0,0,0,0)$. The proof for the remaining $(a, b, c, d) \in \mathcal{L}$ is similar. Comparing the definitions of $\mathcal{H}_{a b c d}$ in Remark 2.4 and of $\mathcal{S}_{a b}^{i j}$ and $\mathbf{y}_{a b c d}$ of Notation 2.5 it follows that

$$
\mathcal{S}_{11}^{01} \cup \mathcal{S}_{11}^{02} \cup \mathcal{S}_{11}^{03} \cup \mathcal{S}_{11}^{12} \cup \mathcal{S}_{11}^{13} \cup \mathcal{S}_{11}^{23} \cup\left\{\mathbf{y}_{1111}\right\} \subset \mathcal{H}_{0000} .
$$

Conversely, let $\mathbf{x} \in \mathcal{H}_{0000}$. From the cubic equations (2.4) involving $y_{0000}$, we see that there exist distinct $i, j \in\{0,1,2,3\}$ such that $x_{i 1}=x_{j 1}=0$. Assume that $i=0$ and $j=1$. If $y_{a b c d}=0$, for all $(a, b, c, d) \in \mathcal{L} \backslash\{(0,0,0,0),(1,1,0,0)\}$, then $y_{0000} y_{1100}-x_{21}^{2} x_{31}^{2}=0$ is the only equation of $Y$ not made trivial. In this situation $\mathbf{x} \in \mathcal{S}_{11}^{23}$. Suppose that $y_{a b c d} \neq 0$ for some $(a, b, c, d) \in \mathcal{L} \backslash$ $\{(0,0,0,0),(1,1,0,0)\}$. Then, from the quartic equations (2.5) involving $y_{a b c d}$ we see that all other weight 2 variables are zero and, using the cubic equations (2.4) involving $y_{a b c d}$, that $x_{2 c}=x_{3 d}=0$. Note that necessarily $(c, d) \neq(0,0)$. Now, if $(c, d)=(1,0)$ then all variables but $y_{a b 10}$ and $x_{31}$ vanish. In this case, either $(a, b)=(0,1)$ and $\mathbf{x} \in \mathcal{S}_{11}^{03}$, or $(a, b)=(1,0)$ and $\mathbf{x} \in \mathcal{S}_{11}^{13}$. Similarly, if $(c, d)=(0,1), \mathbf{x} \in \mathcal{S}_{11}^{02} \cup \mathcal{S}_{11}^{12}$. Finally, if $(c, d)=(1,1)$ then, $\mathbf{x}=\mathbf{y}_{1111}$ or $\mathbf{x}=\mathbf{y}_{0011}$, and we conclude by observing that $\mathbf{y}_{0011} \in \mathcal{S}_{11}^{01}$. The same reasoning applies for any other distinct $i, j \in\{0,1,2,3\}$.

Proposition 2.7. $Y$ is a reduced and irreducible normal 4-dimensional subscheme of $\mathbb{P}\left(1^{8}, 2^{8}\right)$. Moreover $K_{Y}=\mathcal{O}_{Y}(-2)$ and $\operatorname{deg} Y=\operatorname{deg} X+4=12$.

Proof. Let $R$ denote the coordinate ring of $X$. The fact that $\operatorname{dim} Y=4$ is a consequence of the fact that $\operatorname{dim} R_{\mathrm{un}}=\operatorname{dim} R=4$, coming from the general theory of Kustin-Miller unprojection. However it is also a consequence of the isomorphism (2.6) and Lemma 2.6. $R_{\text {un }}$ is obtained as an unprojection of $\mathrm{R}$, that has canonical module equal to $R(-2)$. Hence $R_{\text {un }}$ is Gorenstein and has a canonical module equal to $R_{\text {un }}(-2), c f$. [27]. In view of Remark 2.1, isomorphism (2.6) and Lemma 2.6, codim Sing $Y \geq 2$. Since $R_{\mathrm{un}}$ is Cohen-Macaulay we deduce that $R_{\mathrm{un}}$ is a normal domain, $c f$. [16, Theorem 18.15]. Hence $Y$ is a reduced and irreducible normal subscheme of $\mathbb{P}\left(1^{8}, 2^{8}\right)$. That $K_{Y}=\mathcal{O}_{Y}(-2)$ follows from the computation of the canonical module of $R_{\mathrm{un}}$. By [27, Proposition 3.4], $\operatorname{deg} Y=\operatorname{deg} X+4=12$.

We can now define the key variety $V$. This variety is obtained intersecting $Y$ with the hypersurface given by $x_{00}+x_{01}=0$. The reason for this choice of degree 1 polynomial will be clear from the action of $G \cong(\mathbb{Z} / 2)^{\oplus 3}$ on $V$ that we describe below. We will regard $V$ as a subvariety of $\mathbb{P}\left(1^{8}, 2^{8}\right)$ defined by the ideal $J+\left(x_{00}+x_{01}\right)$, i.e., the ideal generated by $x_{00}+x_{01}$ and the polynomials in (2.1), (2.4) and (2.5). Since $x_{00}+x_{01}$ is a regular element of $R_{\text {un }}$ and this ring is Cohen-Macaulay we deduce that $V$ is a 3 -fold of degree 12 . Clearly, $V$ is the 
parallel unprojection of the 8 planes $\Pi_{a b c d}:=H_{a b c d} \cap\left(x_{00}+x_{11}=0\right)$ in the 3 -fold $W=X \cap\left(x_{00}+x_{01}=0\right)$. The following diagram shows the construction so far.

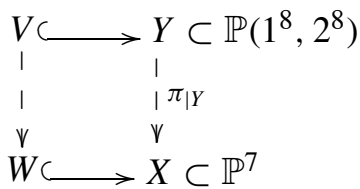

Proposition 2.8. The singular locus of $V=Y \cap\left(x_{00}+x_{01}=0\right)$ consists of 14 points, 8 quotient singularities of type $\frac{1}{2}(1,1,1)$ at the points $\mathbf{y}_{\text {abcd }}$ and 6 isolated singularities locally analytically isomorphic to the vertex of a cone over the Del Pezzo surface $\mathbb{P}^{1} \times \mathbb{P}^{1} \subset \mathbb{P}^{8}$ at the points $\mathbf{x}_{i a} \in \mathbb{P}\left(1^{8}, 2^{8}\right)$, for $i>0$.

Proof. Consider $W=X \cap\left(x_{00}+x_{01}=0\right)$. The variety $W$ is smooth away from $\cup_{a b c d \in \mathcal{L}} H_{a b c d}$. Since $\pi$ is an isomorphism away from $\cup_{a b c d \in \mathcal{L}} \mathcal{H}_{a b c d}(c f$. Remark 2.4), we deduce that

$$
\operatorname{Sing}(V) \subset V \cap\left(\cup_{a b c d \in \mathcal{L}} \mathcal{H}_{a b c d}\right)=V \cap\left(\cup \mathcal{S}_{a b}^{i j}\right)
$$

We start by analyzing the points $\mathbf{x}$ of $\operatorname{Sing}(V)$ in the locus $\left\{\mathbf{y}_{a b c d} \mid(a, b, c, d) \in \mathcal{L}\right\}$. We assume, without loss of generality, that $\mathbf{x}=\mathbf{y}_{0000}$. Consider the affine piece of $V$ given by $y_{0000}=1$. Then, using the quartic equations (2.5) we can eliminate all of the remaining $y_{a b c d}$, using the cubic equations (2.4) we can eliminate $x_{00}, x_{10}, x_{20}, x_{30}$ and using $x_{00}+x_{01}$ we can eliminate $x_{01}$. The coordinates $x_{11}$, $x_{21}, x_{31}, y_{0000}$ map an analytic neighborhood of $\mathbf{y}_{0000} \in V$ isomorphically onto a neighborhood of the point $(0,0,0,1) \in \mathbb{P}\left(1^{3}, 2\right)$, which is a quotient singularity of type $\frac{1}{2}(1,1,1)$.

Suppose now that $\mathbf{x} \in \operatorname{Sing}(V) \backslash\left\{\mathbf{y}_{a b c d} \mid(a, b, c, d) \in \mathcal{L}\right\}$. Let $V_{\mathrm{a}} \subset \mathbb{A}^{16}$ denote the affine cone of $V$. Among the equations of $V_{\mathrm{a}}$, besides $x_{00}+x_{01}=0$, we find the 7 quartic equations $y_{a b c d} y_{0000}-\cdots=0$, plus

$$
\begin{array}{ll}
y_{0000} x_{00}-x_{11} x_{21} x_{31}=0, & y_{0000} x_{10}-x_{01} x_{21} x_{31}=0, \\
y_{0000} x_{20}-x_{01} x_{11} x_{31}=0, & y_{0000} x_{30}-x_{01} x_{11} x_{21}=0
\end{array}
$$

Let us take the $12 \times 12$ minor of the Jacobian matrix of the ideal defining $V_{\mathrm{a}}$ of the gradients of these 12 polynomials with respect to the variables $x_{01}, y_{a b c d}$ for $(a, b, c, d) \in \mathcal{L} \backslash\{(0,0,0,0)\}$ and $x_{00}, x_{10}, x_{20}, x_{30}$. This minor is equal to $\pm y_{0000}^{11}$ where the sign depends on the order we give to the equations and to the variables. Similarly we can find minors of the form $\pm y_{a b c d}^{11}$, for all $(a, b, c, d) \in \mathcal{L}$. Hence if $\mathbf{x} \in \operatorname{Sing}(V) \backslash\left\{\mathbf{y}_{a b c d} \mid(a, b, c, d) \in \mathcal{L}\right\}$ then $y_{a b c d}=0$, for all $(a, b, c, d) \in$ $\mathcal{L}$. From (2.8) and Lemma 2.6, we deduce $\mathbf{x} \in\left\{\mathbf{x}_{10}, \mathbf{x}_{11}, \mathbf{x}_{20}, \mathbf{x}_{21}, \mathbf{x}_{30}, \mathbf{x}_{11}\right\}$. We assume, without loss of generality, that $\mathbf{x}=\mathbf{x}_{10}$. Consider the affine piece of $Y$ given by $x_{10}=1$. Here, we can use the cubic equations (2.4) to eliminate all variables of the form $y_{a 0 c d}$ and one of the quadrics (2.1) to eliminate $x_{11}$. After 
eliminating these 5 variables, we see that this affine piece of $Y$ is isomorphic to the subvariety of $\mathbb{A}^{9}$ defined by the $2 \times 2$ minors of the symmetric matrix

$$
\left(\begin{array}{cccc}
y_{1100} & x_{31} & x_{21} & x_{00} \\
& y_{0110} & x_{01} & x_{20} \\
& & y_{0101} & x_{30} \\
\text { sym } & & & y_{1111}
\end{array}\right)
$$

with $\mathbf{x}_{10}$ being identified with the origin of $\mathbb{A}^{9}$. Hence $\mathbf{x}_{10}$ is a singular point of $Y$ locally isomorphic to the cone over the 2-Veronese embedding of $\mathbb{P}^{3}$ in $\mathbb{P}^{9}$. Since $V=Y \cap\left(x_{00}+x_{01}=0\right)$ we conclude that $V$ is locally, near $\mathbf{x}_{10}$, analytically isomorphic to a cone over the Del Pezzo surface $\mathbb{P}^{1} \times \mathbb{P}^{1} \subset \mathbb{P}^{8}$. Similarly for all other points in $\left\{\mathbf{x}_{10}, \mathbf{x}_{11}, \mathbf{x}_{20}, \mathbf{x}_{21}, \mathbf{x}_{30}, \mathbf{x}_{31}\right\}$.

Corollary 2.9. $V$ is a reduced and irreducible normal 3-dimensional subscheme of $\mathbb{P}\left(1^{8}, 2^{8}\right)$. Moreover $K_{V}=\mathcal{O}_{V}(-1)$ and $\operatorname{deg}(V)=12$.

Proof. The proof is similar to that of Proposition 2.7.

The surface $T$, on which we will set up a group action of $G \cong(\mathbb{Z} / 2)^{3}$ will be a suitable hypersurface section of $V$ of degree 2 , and therefore a canonical surface. In particular the group action is induced by action of $G$ on the ambient weighted projective space. What we do next is to set an action of the larger group $(\mathbb{Z} / 2)^{6}$ on the ambient space, which leaves $V$ invariant. Following that, we single out a subgroup $G \cong(\mathbb{Z} / 2)^{3}$ of $(\mathbb{Z} / 2)^{6}$ inducing on $H^{0}\left(\mathcal{O}_{V}(1)\right)$ the regular representation of $G$ minus the trivial rank 1 representation. Finally, we choose the surface $T \in\left|\mathcal{O}_{V}(2)\right|$ in such a way that $G$ leaves it invariant and that the induced representation of $G$ on $H^{0}\left(\mathcal{O}_{T}(2)\right)=H^{0}\left(K_{T}\right)$ is the sum of 4 copies of the regular representation.

Let $\alpha_{1}, \alpha_{2}, \alpha_{3}, \beta_{1}, \beta_{2}, \beta_{3}$ be generators of $(\mathbb{Z} / 2)^{6}$. Let them act on the space $\left\langle x_{i j}\right\rangle$ in the following way: $\alpha_{i}$ exchanges $x_{00}$ with $x_{01}$ and exchanges $x_{i 0}$ with $x_{i 1}$, fixing all the remaining variables; $\beta_{i}$ takes $x_{i 0}$ to $-x_{i 0}$ and $x_{i 1}$ to $-x_{i 1}$, fixing all the remaining variables. Since the actions of two generators commute, we obtain an action of $(\mathbb{Z} / 2)^{6}$ on $\mathbb{P}^{7}$. Clearly, by inspection of $(2.1), X$ is invariant under this action. The identification of the variables $y_{a b c d}$ with the rational functions on $X$ of (2.3) induces an extension of this action to $\mathbb{P}\left(1^{8}, 2^{8}\right)$ so that $Y$, and $V$ as well, become invariant. Since

$$
\varphi_{a b c d}=\frac{x_{1 b^{\prime}} x_{2 c^{\prime}} x_{3 d^{\prime}}}{x_{0 a}} \stackrel{\alpha_{1}}{\longrightarrow} \frac{x_{1 b} x_{2 c^{\prime}} x_{3 d^{\prime}}}{x_{0 a^{\prime}}}=\varphi_{a^{\prime} b^{\prime} c d},
$$

etc., it suffices to set $\alpha_{1}\left(y_{a b c b d}\right)=y_{a^{\prime} b^{\prime} c d}, \alpha_{2}\left(y_{a b c d}\right)=y_{a^{\prime} b c^{\prime} d}, \alpha_{3}\left(y_{a b c d}\right)=y_{a^{\prime} b c d^{\prime}}$ and $\beta_{i}\left(y_{a b c d}\right)=-y_{a b c d}$, for all $1 \leq i \leq 3$. We summarize this in Table 2.1.

Consider the subgroup $G \subset(\mathbb{Z} / 2)^{6}$ given by

$$
G=\left\langle\alpha_{1} \beta_{2}, \alpha_{2} \beta_{3}, \alpha_{3} \beta_{1}\right\rangle \cong(\mathbb{Z} / 2)^{3} .
$$


Table 2.1. The $(\mathbb{Z} / 2)^{6}$-action.

\begin{tabular}{cccc}
\hline$\alpha_{1}$ & $x_{00} \leftrightarrow x_{01}$ & $x_{10} \leftrightarrow x_{11}$ & $y_{a b c d} \leftrightarrow y_{a^{\prime} b^{\prime} c d}$ \\
\hline$\alpha_{2}$ & $x_{00} \leftrightarrow x_{01}$ & $x_{20} \leftrightarrow x_{21}$ & $y_{a b c d} \leftrightarrow y_{a^{\prime} b c^{\prime} d}$ \\
\hline$\alpha_{3}$ & $x_{00} \leftrightarrow x_{01}$ & $x_{30} \leftrightarrow x_{31}$ & $y_{a b c d} \leftrightarrow y_{a^{\prime} b c d^{\prime}}$ \\
\hline$\beta_{i}$ & $x_{i 0} \rightarrow-x_{i 0}$ & $x_{i 1} \rightarrow-x_{i 1}$ & $y_{a b c d} \rightarrow-y_{a b c d}$ \\
\hline
\end{tabular}

It is easy to see that the representation of $G$ on $H^{0}\left(\mathcal{O}_{V}(1)\right)$ is the regular representation minus the trivial rank 1 representation; indeed the representation of $G$ on $\left\langle x_{00}, x_{01}, \ldots, x_{31}\right\rangle$ is the regular representation and $x_{00}+x_{01}$ generates the invariant eigenspace. Likewise, given a character $\epsilon \in \operatorname{Hom}\left((\mathbb{Z} / 2)^{3}, \mathbb{C}\right)$, it is not hard to see that the polynomial

$$
\sum_{a b c d \in \mathcal{L}} \epsilon(b, c, d) y_{a b c d}
$$

is an eigenvector for the action of $G$ on the space $\left\langle y_{a b c d} \mid(a, b, c, d) \in \mathcal{L}\right\rangle$ and that the 8 polynomials obtained in this way generate distinct eigenspaces of the action. The expression for the trivial eigenvector, obtained from (2.11) using the character given by $\epsilon(b, c, d)=(-1)^{b+c+d}$, for all $(b, c, d) \in(\mathbb{Z} / 2)^{3}$, is given by:

$$
\sum_{a b c d \in \mathcal{L}}(-1)^{b+c+d} y_{a b c d}=\sum_{a b c d \in \mathcal{L}}(-1)^{a} y_{a b c d} .
$$

The representation theory of $G$ on the cohomology of $T$ dictates the eigenspace of $H^{0}\left(\mathcal{O}_{V}(2)\right)$ from which to take the equation of $T \in\left|\mathcal{O}_{V}(2)\right|$. According to (1.1) and the discussion above, the equation for $T$ belongs to the invariant eigenspace of $H^{0}\left(\mathcal{O}_{V}(2)\right)$. Consider the following invariant quadratic forms in the $x_{i a}$ variables:

$$
s_{i}=\frac{x_{i 0}^{2}+x_{i 1}^{2}}{2} \text { and } t_{i}=x_{i 0} x_{i 1} \text { for } i=0,1,2,3 .
$$

using $x_{00}+x_{01}=0$ and (2.1) we obtain

$$
t_{i}=t_{0}=-s_{0}
$$

on $V$ and $W$. Hence $s_{0}, s_{1}, s_{2}, s_{3}$ form a basis for the invariant subspace of the second symmetric power of $H^{0}\left(\mathcal{O}_{V}(1)\right)$. From this and (2.12) we see that a general element of the invariant eigenspace of $H^{0}\left(\mathcal{O}_{V}(2)\right)$ is given by:

$$
q=l+v_{4} \sum_{a b c d \in \mathcal{L}}(-1)^{a} y_{a b c d}, \quad \text { where } \quad l=v_{0} s_{0}+v_{1} s_{1}+v_{2} s_{2}+v_{3} s_{3}
$$

and $v_{0}, v_{1}, v_{2}, v_{3}, v_{4}$ are general complex parameters. Let $\mathcal{N} \cong \mathbb{P}^{4}$ be the linear system of surfaces given by

$$
\mathcal{N}=\left\{T=V \cap(q=0) \mid\left(v_{0}, v_{1}, v_{2}, v_{3}, v_{4}\right) \in \mathbb{P}^{4}\right\} .
$$


Then $G$ acts on every $T \in \mathcal{N}$, and we can take the quotient $S=T / G$.

Theorem 2.10. A general element $T \in \mathcal{N}$ is smooth surface of general type with ample canonical divisor and with $p_{g}(T)=7, q(T)=0$ and $K_{T}^{2}=24$. Furthermore the canonical map of $T$ is a birational morphism onto a complete intersection of three quadrics and a cubic in $\mathbb{P}^{6}$. For a general surface $T \in \mathcal{N}$, the action of $G$ is free and therefore $S:=T / G$ is a surface of general type with ample canonical divisor and with $p_{g}(S)=0$ and $K_{S}^{2}=3$.

Proof. The base locus of $\mathcal{N}$ is contained in the locus given by $\left(s_{0}=s_{1}=s_{2}=\right.$ $\left.s_{3}=0\right)$. Using (2.13) and (2.14), we get $x_{i 0} x_{i 1}=0$ for all $i=0,1,2,3$; and since $2 s_{i}=x_{i 0}^{2}+x_{i 1}^{2}$ we deduce $x_{i 0}=x_{i 1}=0$ for all $i=0,1,2,3$. Therefore

$$
\left(s_{0}=s_{1}=s_{2}=s_{3}=0\right) \cap V=\left\{\mathbf{y}_{a b c d} \mid(a, b, c, d) \in \mathcal{L}\right\},
$$

which, for general $v_{0}, v_{1}, v_{2}, v_{3}$, does not intersect $T$. By Bertini's Theorem, $\operatorname{Sing}(T)$ is contained in the union of the base locus of $\mathcal{N}$ and $\operatorname{Sing}(V)$. For a general choice of $v_{0}, v_{1}, v_{2}, v_{3}, v_{4}$, the surface $T$ does not meet $\operatorname{Sing}(V)$, ( $c f$. Proposition 2.8), and as we showed, $\mathcal{N}$ is base point free. Hence $T$ is nonsingular. Since the coordinate ring of $T$ is the quotient of $R_{\text {un }}$ by a regular sequence, it is a Gorenstein graded ring and, in particular, Cohen-Macaulay. By [16, Theorem 18.15] the coordinate ring of $T$ is a domain and, accordingly, $T$ is reduced and irreducible. By adjunction, $K_{T}=\mathcal{O}_{T}(1)$ which is ample, and the projectively Gorensteinness of $T$ yields $q=\operatorname{dim} H^{1}\left(K_{T}\right)=0$ and $p_{g}(T)=7$. Finally $K_{T}^{2}=\operatorname{deg}(T)=2 \operatorname{deg}(V)=24$.

The canonical map $\varphi_{K_{T}}$ of $T$ equals $\pi_{\mid T}$, the map given by the sections $x_{00}=$ $x_{01}, x_{10}, x_{11}, x_{20}, x_{21}, x_{30}, x_{31}, c f$. Notation 2.3. Since the locus of common zeros of these sections is contained in the locus $\left(s_{0}=s_{1}=s_{2}=s_{3}=0\right)$ we deduce that $\varphi_{K_{T}}$ is a morphism. Moreover since $K_{T}$ is ample, $\varphi_{K_{T}}$ is finite. Since $\pi_{\mid V}$ is birational, and $T \in \mathcal{N}$ is a general element of a movable linear system, $\varphi_{K_{T}}$ is also birational. Then the canonical image $\varphi_{K_{T}}(T)$ is a nondegenerate surface of degree $K_{T}^{2}=24$ in the hyperplane $\mathbb{P}^{6}:=\left(x_{00}+x_{01}=0\right) \subset \mathbb{P}^{7}$, contained in the locus defined by (2.1). By elimination, we find a new cubic hypersurface through $\varphi_{K_{T}}(T)$. From $q=0 \Longleftrightarrow v_{4} \sum_{a b c d \in \mathcal{L}}(-1)^{a} y_{a b c d}=-l$, substituting $y_{a b c d}$ with $\frac{x_{1 b^{\prime}} x_{2 c^{\prime}} x_{3 d^{\prime}}}{x_{0 a}}$ and using $x_{01}=-x_{00}$ we get

$$
v_{4} \sum_{a b c d \in \mathcal{L}}(-1)^{a} \frac{x_{1 b^{\prime}} x_{2 c^{\prime}} x_{3 d^{\prime}}}{x_{0 a}}=l \Longleftrightarrow v_{4} \sum_{b c d \in\{0,1\}^{3}} \frac{x_{1 b^{\prime}} x_{2 c^{\prime}} x_{3 d^{\prime}}}{x_{00}}=l,
$$

which yields the irreducible cubic equation:

$$
v_{4}\left(x_{10}+x_{11}\right)\left(x_{20}+x_{21}\right)\left(x_{30}+x_{31}\right)=x_{00} l \text {. }
$$

Therefore $\varphi_{K_{T}}(T)$ is a surface of degree 24 contained in the intersection of the hyperplane $\left(x_{00}+x_{01}=0\right)$, the quadrics (2.1) and the cubic defined by (2.17). Since these polynomials form a regular sequence, we deduce that $\varphi_{K_{T}}(T)$ coincides 
with the complete intersection of 3 quadrics and 1 cubic that, choosing $x_{00}, x_{10}$, $x_{11}, \ldots, x_{30}, x_{31}$ as basis for $H^{0}\left(K_{T}\right)$, are obtained substituting $x_{01}$ for $-x_{00}$ in (2.1) and (2.17).

Let us now show that the action of $G$ on $T$ is free. By symmetry it is enough to check that the 3 elements $\alpha_{1} \beta_{2}, \alpha_{1} \alpha_{2} \beta_{2} \beta_{3}$ and $\alpha_{1} \alpha_{2} \alpha_{3} \beta_{1} \beta_{2} \beta_{3}$ act on $T$ without fixed points. In the weighted projective space $\mathbb{P}\left(1^{8}, 2^{8}\right)$ the fixed locus of an involution splits into three spaces; the $(+,+)$ part $(i . e .$, positive on the $x$ variables and positive on the $y$ variables), the $(-,+)$ part and the $(0,-)$ part (i.e., negative on the $y$ variables with all the $x$ variables 0 ); since the last space cuts out the empty set on $T$, we will repeatedly ignore it. Denote these spaces by $\operatorname{Fix}_{(+,+)}$and Fix $(-,+)$. Then, referring to Table 2.1 , we see that $\operatorname{Fix}_{(+,+)}\left(\alpha_{1} \beta_{2}\right)$ is equal to:

$$
\left(x_{00}-x_{01}=x_{10}-x_{11}=x_{20}=x_{21}=y_{a b c d}+y_{a^{\prime} b^{\prime} c d}=0, \forall_{a b c d \in \mathcal{L}}\right) \text {. }
$$

From (2.1) we get $x_{00} x_{01}=x_{10} x_{11}=0$ and hence $x_{00}=x_{01}=x_{10}=x_{11}=0$. Thus all coordinates $x_{i a}$ vanish except for, possibly, $x_{30}$ or $x_{31}$. From the quartic relation $y_{a b c d} y_{a^{\prime} b^{\prime} c d}=x_{2 c^{\prime}}^{2} x_{3 d^{\prime}}^{2}=0, c f$. (2.5), and $y_{a b c d}+y_{a^{\prime} b^{\prime} c d}=0$ we deduce that $y_{a b c d}=0$ for all $(a, b, c, d) \in \mathcal{L}$. Using $q=0$ we obtain $x_{30}=x_{31}=0$. Hence $T$ does not meet $\operatorname{Fix}_{(+,+)}\left(\alpha_{1} \beta_{2}\right)$.

Next we consider the loci $\operatorname{Fix}_{(-,+)}\left(\alpha_{1} \beta_{2}\right), \operatorname{Fix}_{(+,+)}\left(\alpha_{1} \alpha_{2} \beta_{2} \beta_{3}\right), \operatorname{Fix}_{(-,+)}\left(\alpha_{1} \alpha_{2} \beta_{2} \beta_{3}\right)$ and $\operatorname{Fix}_{(+,+)}\left(\alpha_{1} \alpha_{2} \alpha_{3} \beta_{1} \beta_{2} \beta_{3}\right)$ which are given by:

$$
\begin{gathered}
\left(x_{00}+x_{01}=x_{10}+x_{11}=x_{30}=x_{31}=y_{a b c d}+y_{a^{\prime} b^{\prime} c d}=0, \forall_{a b c d \in \mathcal{L}}\right), \\
\left(x_{10}-x_{11}=x_{20}+x_{21}=x_{30}=x_{31}=y_{a b c d}-y_{a b^{\prime} c^{\prime} d}=0, \forall_{a b c d \in \mathcal{L}}\right), \\
\left(x_{00}=x_{01}=x_{10}+x_{11}=x_{20}-x_{21}=y_{a b c d}-y_{a b^{\prime} c^{\prime} d}=0, \forall_{a b c d \in \mathcal{L}}\right), \\
\left(x_{00}-x_{01}=x_{10}+x_{11}=x_{20}+x_{21}=x_{30}+x_{31}=y_{a b c d}+y_{a^{\prime} b^{\prime} c^{\prime} d^{\prime}}=0, \forall a b c d \in \mathcal{L}\right),
\end{gathered}
$$

respectively. Arguing as before (remembering, for the last locus, that $x_{00}+x_{01}=0$ holds) we see that none of them meets $T$.

Finally $\operatorname{Fix}_{(-,+)}\left(\alpha_{1} \alpha_{2} \alpha_{3} \beta_{1} \beta_{2} \beta_{3}\right)$ is given by:

$$
\left(x_{00}+x_{01}=x_{10}-x_{11}=x_{20}-x_{21}=x_{30}-x_{31}=y_{a b c d}+y_{a^{\prime} b^{\prime} c^{\prime} d^{\prime}}=0, \forall_{a b c d \in \mathcal{L}}\right) \text {. }
$$

Using (2.1) we get $x_{j 0}^{2}=-x_{00}^{2}$. Hence $s_{j}=-s_{0}$, for all $j=1,2,3$. From the quartic equations (2.5) we get $-y_{a b c d}^{2}=y_{a b c d} y_{a^{\prime} b^{\prime} c^{\prime} d^{\prime}}=x_{20} x_{21} x_{30} x_{31}=x_{00}^{4}$. Taking square roots of this equation, substituting in $q=0$ and using the generality of $v_{0}, v_{1}, v_{2}, v_{3}, v_{4}$ we deduce that $x_{00}=0$; and hence $x_{01}=x_{j 0}=$ $x_{j 1}=0$ for all $j=1,2,3$ and $y_{a b c d}=0$ for all $(a, b, c, d) \in \mathcal{L}$. Therefore $T \cap \operatorname{Fix}_{(-,+)}\left(\alpha_{1} \alpha_{2} \alpha_{3} \beta_{1} \beta_{2} \beta_{3}\right)=\emptyset$.

Since the action of $G$ on $T$ is free, $S=T / G$ is a nonsingular surface of general type with $p_{g}(S)=0$ and $K_{S}^{2}=3$. Since $K_{T}$ is ample, we deduce that $K_{S}$ is ample. 
Remark 2.11. Theorem 2.10 shows that for every $T \in \mathcal{N}$ such that

- $T$ has at most canonical singularities,

- the action of $G$ on $T$ is free,

the quotient $S=T / G$ is the canonical model of a surface of general type with $p_{g}(S)=0$ and $K^{2}=3$ : this provides a 4-dimensional family of these surfaces.

Remark 2.12. By analysis of the proof of Theorem 2.10 , we see that if, for a given $T \in \mathcal{N}$, the action of $G$ has any fixed points on $T$ then either $v_{1} v_{2} \nu_{3}=0$ or there exists $\delta$ in a finite set of (integer) multiples of $i$ such that $v_{0}-v_{1}-v_{2}-v_{3}+\delta v_{4}=0$. We shall use this observation later on.

\section{A double cover}

Consider the Fano 4-fold $\mathbb{P}^{1} \times \mathbb{P}^{1} \times \mathbb{P}^{1} \times \mathbb{P}^{1}$ with coordinates $\left(t_{00}, t_{01}\right),\left(t_{10}, t_{11}\right)$, $\left(t_{20}, t_{21}\right),\left(t_{30}, t_{31}\right)$, and let $\sigma: \mathbb{P}^{1} \times \mathbb{P}^{1} \times \mathbb{P}^{1} \times \mathbb{P}^{1} \rightarrow \mathbb{P}\left(1^{8}, 2^{8}\right)$ be the map given by:

$$
\begin{gathered}
\sigma^{\sharp}\left(x_{0 a}\right)=t_{0 a^{\prime}} t_{1 a} t_{2 a} t_{3 a}, \sigma^{\sharp}\left(x_{1 a}\right)=t_{0 a} t_{1 a^{\prime}} t_{2 a} t_{3 a}, \\
\sigma^{\sharp}\left(x_{2 a}\right)=t_{0 a} t_{1 a} t_{2 a^{\prime}} t_{3 a}, \sigma^{\sharp}\left(x_{3 a}\right)=t_{0 a} t_{1 a} t_{2 a} t_{3 a^{\prime}}, \\
\sigma^{\sharp}\left(y_{a b c d}\right)=t_{0 a^{\prime}}^{2} t_{1 b^{\prime}}^{2} t_{2 c^{\prime}}^{2} t_{3 d^{\prime}}^{2}, \text { if } a=b=c=d \text { and } \sigma^{\sharp}\left(y_{a b c d}\right)=t_{0 a}^{2} t_{1 b}^{2} t_{2 c}^{2} t_{3 d}^{2} \text { otherwise. }
\end{gathered}
$$

It is straightforward to check that $\sigma\left(\mathbb{P}^{1} \times \mathbb{P}^{1} \times \mathbb{P}^{1} \times \mathbb{P}^{1}\right)=Y \subset \mathbb{P}\left(1^{8}, 2^{8}\right)$.

Proposition 3.1. The map $\sigma: \mathbb{P}^{1} \times \mathbb{P}^{1} \times \mathbb{P}^{1} \times \mathbb{P}^{1} \rightarrow Y$ is finite of degree 2 branched exactly at the set $\left\{\mathbf{x}_{i a}, \mathbf{y}_{a b c d}: 0 \leq i \leq 3,(a, b, c, d) \in \mathcal{L}\right\}$.

Proof. Let $U_{i a} \subset Y$ be the open subset of $Y$ given by $x_{i a} \neq 0$. First note that $\sigma^{-1}\left(\mathbf{y}_{a b c d}\right)$ consists of a point, more precisely one of the coordinate points of $\mathbb{P}^{1} \times$ $\mathbb{P}^{1} \times \mathbb{P}^{1} \times \mathbb{P}^{1}$. Moreover, the family $\left\{U_{i a}\right\}$, with $0 \leq i \leq 3$ and $a \in\{0,1\}$ is an open affine cover of $Y \backslash\left\{\mathbf{y}_{a b c d}\right\}$. Consider the restriction $\sigma_{\mid}: \sigma^{-1}\left(U_{00}\right) \rightarrow U_{00}$. The open set $\sigma^{-1}\left(U_{00}\right)$ is simply $\mathbb{C}^{4}$ with coordinates

$$
\frac{t_{00}}{t_{01}}, \frac{t_{11}}{t_{10}}, \frac{t_{21}}{t_{20}}, \frac{t_{31}}{t_{30}}
$$

The coordinate ring of $U_{00}$, which we denote by $\mathbb{C}\left[U_{00}\right]$, is generated by the regular functions:

$$
\frac{x_{i a}}{x_{00}}, \quad \frac{y_{a b c d}}{x_{00}^{2}}, \quad \text { with } 0 \leq i \leq 3 \text { and }(a, b, c, d) \in \mathcal{L}
$$

Computing the image by $\sigma_{\mid}^{\sharp}$ of each of the generators of $\mathbb{C}\left[U_{00}\right]$, we get the generators of the ideal $\left(\frac{t_{00}}{t_{01}}, \frac{t_{11}}{t_{10}}, \frac{t_{21}}{t_{20}}, \frac{t_{31}}{t_{30}}\right)^{2}$. Hence $\sigma_{1}: \sigma^{-1}\left(U_{00}\right) \rightarrow U_{00}$ is finite of 
degree 2. The same computation on each $U_{i a}$ yields the same result, showing that $\sigma$ is a double cover. Additionally, the involution $s \in \operatorname{Aut}\left(\mathbb{P}^{1} \times \mathbb{P}^{1} \times \mathbb{P}^{1} \times \mathbb{P}^{1}\right)$ given by

$$
s\left(t_{i a}\right)=(-1)^{a} t_{i a}, \quad \text { for } 0 \leq i \leq 3 \text { and } a \in\{0,1\}
$$

satisfies $\sigma \circ s=\sigma$. Note that $s$ has exactly 16 fixed points, the coordinate points of $\mathbb{P}^{1} \times \mathbb{P}^{1} \times \mathbb{P}^{1} \times \mathbb{P}^{1}$. In particular, $\sigma$ branches exactly at their images, i.e. the points in the set $\left\{\mathbf{x}_{i a}, \mathbf{y}_{a b c d}: 0 \leq i \leq 3,(a, b, c, d) \in \mathcal{L}\right\}$.

Remark 3.2. We can deduce from Proposition 3.1 that Sing $Y$ is the set of 16 points $\left\{\mathbf{x}_{i a}, \mathbf{y}_{a b c d}: 0 \leq i \leq 3,(a, b, c, d) \in \mathcal{L}\right\}$, which are quotient singularities of type $\frac{1}{2}(1,1,1,1)$. This agrees with Proposition 2.8 .

Remark 3.3. The restriction of $\sigma$ to the Fano 3-fold

$$
Z_{1}=\left(t_{01} t_{10} t_{20} t_{30}+t_{00} t_{11} t_{21} t_{31}=0\right) \subset \mathbb{P}^{1} \times \mathbb{P}^{1} \times \mathbb{P}^{1} \times \mathbb{P}^{1}
$$

is a double cover of $V$, branched on the 14 singularities of $V$. The 3-fold $Z_{1}$ is a (special) member of $|\mathcal{O}(1,1,1,1)|^{-}$, the linear system of effective divisors on $\mathbb{P}^{1} \times \mathbb{P}^{1} \times \mathbb{P}^{1} \times \mathbb{P}^{1}$ of degree $(1,1,1,1)$ anti-invariant respect to the involution $s$. A general member of $|\mathcal{O}(1,1,1,1)|^{-}$is the canonical double cover of an EnriquesFano 3-fold with only terminal singularities. These 3-folds were classified by Bayle and Sano $[10,33]$. The image of a general member of $|\mathcal{O}(1,1,1,1)|^{-}$under $\sigma$ falls in case 10 of Sano's list. Indeed the whole construction in this section has been inspired by that case.

Recall that $(\mathbb{Z} / 2)^{6}$ acts on $Y$ as given in Table 2.1 .

Table 3.1. Automorphisms of $\mathbb{P}^{1} \times \mathbb{P}^{1} \times \mathbb{P}^{1} \times \mathbb{P}^{1}$. (For the last 4 , since the action is diagonal we list only the eigenvalues. Here $\epsilon$ is a square-root of -1 .)

\begin{tabular}{ccccccccc}
\hline & $t_{00}$ & $t_{01}$ & $t_{10}$ & $t_{11}$ & $t_{20}$ & $t_{21}$ & $t_{30}$ & $t_{31}$ \\
\hline$\tilde{\alpha}_{1}$ & $t_{10}$ & $t_{11}$ & $t_{00}$ & $t_{01}$ & $t_{31}$ & $t_{30}$ & $t_{21}$ & $t_{20}$ \\
\hline$\tilde{\alpha}_{2}$ & $t_{20}$ & $t_{21}$ & $t_{31}$ & $t_{30}$ & $t_{00}$ & $t_{01}$ & $t_{11}$ & $t_{10}$ \\
\hline$\tilde{\alpha}_{3}$ & $t_{30}$ & $t_{31}$ & $t_{21}$ & $t_{20}$ & $t_{11}$ & $t_{10}$ & $t_{00}$ & $t_{01}$ \\
\hline$\tilde{\beta}_{1}$ & $-\epsilon$ & 1 & 1 & $-\epsilon$ & 1 & $\epsilon$ & 1 & $\epsilon$ \\
\hline$\tilde{\beta}_{2}$ & $-\epsilon$ & 1 & 1 & $\epsilon$ & 1 & $-\epsilon$ & 1 & $\epsilon$ \\
\hline$\tilde{\beta}_{3}$ & $-\epsilon$ & 1 & 1 & $\epsilon$ & 1 & $\epsilon$ & 1 & $-\epsilon$ \\
\hline$s$ & 1 & -1 & 1 & -1 & 1 & -1 & 1 & -1 \\
\hline
\end{tabular}

In Table 3.1, we distinguish a set of automorphisms of $\mathbb{P}^{1} \times \mathbb{P}^{1} \times \mathbb{P}^{1} \times \mathbb{P}^{1}$, one of which, $s$, has already been defined in (3.1) and the remaining ones are meant to lift the actions of $\alpha_{1}, \alpha_{2}, \alpha_{3}, \beta_{1}, \beta_{2}, \beta_{3}$. One can check by direct computation that $\tilde{\alpha}_{1}, \tilde{\alpha}_{2}, \tilde{\alpha}_{3}, \tilde{\beta}_{1}, \tilde{\beta}_{2}, \tilde{\beta}_{3}$ lift the action of $\alpha_{1}, \alpha_{2}, \alpha_{3}, \beta_{1}, \beta_{2}, \beta_{3}$, i.e., that $\sigma \circ \tilde{\alpha}_{i}=$ $\alpha_{1} \circ \sigma$ and $\sigma \circ \tilde{\beta}_{i}=\beta_{1} \circ \sigma$, for $i=1,2,3$. On the other hand, there are a number of small checks that are straightforward. It is clear that $s$ commutes with every other automorphism listed in Table 3.1 ; it is also clear that $\tilde{\alpha}_{1}, \tilde{\alpha}_{2}, \tilde{\alpha}_{3}$ are 
automorphisms of order 2 commuting with each other, that $\tilde{\beta}_{1}, \tilde{\beta}_{2}, \tilde{\beta}_{3}$ commute with each other and that $\tilde{\beta}_{1}^{2}=\tilde{\beta}_{2}^{2}=\tilde{\beta}_{3}^{2}=s$. Finally, a less straightforward (but still elementary) computation shows that $\tilde{\alpha}_{i} \tilde{\beta}_{j}=s^{\delta_{i j}} \tilde{\beta}_{j} \tilde{\alpha}_{i}$, where $\delta_{i j}$ is Kronecker's delta. These identities are useful in the proof of the next proposition, where we characterize the group $\tilde{G}$ generated by the automorphisms that lift the generators of $G=\left\langle\alpha_{1} \beta_{2}, \alpha_{2} \beta_{3}, \alpha_{3} \beta_{1}\right\rangle \simeq(\mathbb{Z} / 2)^{3}$.

Lemma 3.4. $\tilde{G}:=\left\langle\tilde{\alpha}_{1} \tilde{\beta}_{2}, \tilde{\alpha}_{2} \tilde{\beta}_{3}, \tilde{\alpha}_{3} \tilde{\beta}_{1}\right\rangle$ is isomorphic to $\mathbb{Z} / 2 \times Q_{8}$, where $Q_{8}$ is the classical quaternion group.

Proof. Since $\operatorname{deg} \sigma=2,|\tilde{G}|$ equals either $2|G|$, if $s \in \tilde{G}$, or $|G|$, if $s \notin \tilde{G}$. Since $\left(\tilde{\alpha_{1}} \tilde{\beta}_{2}\right)^{2}=s$, we get $|\tilde{G}|=2|G|=16$. Consider the standard presentation of $Q_{8}$ given by

$$
\left\langle-1, i, j, k \mid(-1)^{2}=1, i^{2}=j^{2}=k^{2}=i j k=-1\right\rangle
$$

and, for clarity, let us use multiplicative notation for $\mathbb{Z} / 2=\{1,-1\}$. Set:

$$
\begin{gathered}
\mu(1,-1)=s, \quad \mu(-1,1)=\tilde{\alpha}_{1} \tilde{\beta}_{2} \tilde{\alpha}_{2} \tilde{\beta}_{3} \tilde{\alpha}_{3} \tilde{\beta}_{1}, \\
\mu(1, i)=\tilde{\alpha}_{2} \tilde{\beta}_{3} \tilde{\alpha}_{3} \tilde{\beta}_{1}, \quad \mu(1, j)=\tilde{\alpha}_{3} \tilde{\beta}_{1} \tilde{\alpha}_{1} \tilde{\beta}_{2}, \quad \mu(1, k)=\tilde{\alpha}_{1} \tilde{\beta}_{2} \tilde{\alpha}_{2} \tilde{\beta}_{3} .
\end{gathered}
$$

Using the identities stated earlier, one can check easily that these definitions respect all the relations of $(\mathbb{Z} / 2) \times Q_{8}$ and therefore determine a group homomorphism $\mu:(\mathbb{Z} / 2) \times Q_{8} \rightarrow \tilde{G}$. Since:

$$
\begin{aligned}
& \mu(-1,-i)=\mu(-1,1) \mu(1, i)^{-1}=\tilde{\alpha}_{1} \tilde{\beta}_{2} \tilde{\alpha}_{2} \tilde{\beta}_{3} \tilde{\alpha}_{3} \tilde{\beta}_{1} \tilde{\beta}_{1}^{-1} \tilde{\alpha}_{3} \tilde{\beta}_{3}^{-1} \tilde{\alpha}_{2}=\tilde{\alpha}_{1} \tilde{\beta}_{2}, \\
& \mu(-1,-j)=\left(\tilde{\alpha}_{1} \tilde{\beta}_{2}\right) \tilde{\alpha}_{2} \tilde{\beta}_{3} \tilde{\alpha}_{3} \tilde{\beta}_{1}\left(\tilde{\beta}_{2}^{-1} \tilde{\alpha}_{1}\right) \tilde{\beta}_{1}^{-1} \tilde{\alpha}_{3}=\tilde{\alpha}_{2} \tilde{\beta}_{3} \tilde{\alpha}_{3} \tilde{\beta}_{1} \tilde{\beta}_{1}^{-1} \tilde{\alpha}_{3}=\tilde{\alpha}_{2} \tilde{\beta}_{3}, \\
& \mu(-1,-k)=\tilde{\alpha}_{1} \tilde{\beta}_{2}\left(\tilde{\alpha}_{2} \tilde{\beta}_{3}\right) \tilde{\alpha}_{3} \tilde{\beta}_{1}\left(\tilde{\beta}_{3}^{-1} \tilde{\alpha}_{2}\right) \tilde{\beta}_{2}^{-1} \tilde{\alpha}_{1}=s \tilde{\alpha}_{1} \tilde{\beta}_{2} \tilde{\alpha}_{3} \tilde{\beta}_{1} \tilde{\beta}_{2}^{-1} \tilde{\alpha}_{1}=\tilde{\alpha}_{3} \tilde{\beta}_{1},
\end{aligned}
$$

we deduce that $\mu$ is surjective, which, as $|\tilde{G}|=\left|(\mathbb{Z} / 2) \times Q_{8}\right|$, implies that $\mu$ is an isomorphism.

We can now give a good description of the family of surfaces $T / G$, for general $T$ in the linear system $\mathcal{N}$.

Theorem 3.5. Let $T \in \mathcal{N}$ be a surface with at most canonical singularities for which the action of $G$ on it is free. Then $\pi_{1}(T / G) \cong \mathbb{Z} / 2 \times Q_{8}$ and the universal cover of $T$ is a complete intersection of the two hypersurfaces in $\mathbb{P}^{1} \times \mathbb{P}^{1} \times \mathbb{P}^{1} \times \mathbb{P}^{1}$, $Z_{1}$ and $Z_{2}$, of multi-degrees $(1,1,1,1)$ and $(2,2,2,2)$, respectively, given by:

$$
\begin{aligned}
& Z_{1}=\left(t_{01} t_{10} t_{20} t_{30}+t_{00} t_{11} t_{21} t_{31}=0\right) \text { and } \\
& Z_{2}=\sum_{i=0}^{3} v_{i}\left(t_{i 0}^{2} \prod_{j \neq i} t_{j 1}^{2}+t_{i 1}^{2} \prod_{j \neq i} t_{j 0}^{2}\right)-2 v_{4} \sum_{a b c d \in \mathcal{L}}(-1)^{\frac{b+c+d-a}{2}} t_{0 a}^{2} t_{1 b}^{2} t_{2 c}^{2} t_{3 d}^{2}=0 .
\end{aligned}
$$


Proof. We note that $T$ does not contain any of the 16 points in the set

$$
\left\{\mathbf{x}_{i a}, \mathbf{y}_{a b c d} \mid 0 \leq i \leq 3,(a, b, c, d) \in \mathcal{L}\right\}
$$

Indeed $T$ is a Cartier divisor in $V$, which contains 14 of these points that, by Proposition 2.8, are singular points of $V$ with Zariski tangent space of dimension 5 or 8 . In particular, if $T$ contains one of these points, the Zariski tangent space of $T$ at this point has at least dimension 4 , whereas every canonical singularity of a surface has Zariski tangent space of dimension 3. Since $T$ is the complete intersection of two divisors ( $V$ and a quadric section, given by $x_{00}+x_{01}=0$ and (2.15), respectively) in $Y$, the surface $\tilde{T}:=\sigma^{-1}(T)$ is the complete intersection of their pull-back to $\mathbb{P}^{1} \times \mathbb{P}^{1} \times \mathbb{P}^{1} \times \mathbb{P}^{1}$, which one easily sees are the hypersurfaces $Z_{1}$ and $Z_{2}$, respectively, of the statement of this theorem. By the Lefschetz hyperplane section theorem, $\pi_{1}(\tilde{T})=0$. Now as the composition $\tilde{T} \stackrel{\sigma_{\mid}}{\rightarrow} T \rightarrow S$ is étale, we conclude that $\tilde{T}$ is the universal cover of $S$. In particular, $\pi_{1}(S)$ is isomorphic to the group of automorphisms of the cover, which coincides with the group of automorphisms of $\tilde{T}$ lifting the action of $G$. This is $\tilde{G}$, which, by Lemma 3.4, is isomorphic to $\mathbb{Z} / 2 \times Q_{8}$.

We conclude this section by studying the locus of the moduli space of the surfaces of general type described by the surfaces $S$.

Theorem 3.6. Let $\mathcal{U}$ be the dense open set of $\mathcal{N} \cong \mathbb{P}^{4}$ consisting of the surfaces $T$ with at most canonical singularities on which $G$ acts freely. Then, the map associating to each point of $\mathcal{U}$ the class of the surface $S / G$, in the Gieseker moduli space of surfaces of general type with $\chi=1$ and $K^{2}=3$, is finite. In particular, its image is 4-dimensional and unirational.

Proof. Let $S_{1}:=T_{1} / G, S_{2}:=T_{2} / G$ be surfaces with $T_{1}, T_{2} \in \mathcal{U}$. Assume that $S_{1} \cong S_{2}$. By Theorem 3.5, $\pi_{1}\left(S_{1}\right) \cong \pi_{1}\left(S_{2}\right) \cong(\mathbb{Z} / 2) \times Q_{8}$. Since the Abelianization of $(\mathbb{Z} / 2) \times Q_{8}$ is $(\mathbb{Z} / 2)^{3}$, each $S_{i}$ has exactly one $(\mathbb{Z} / 2)^{3}$-cover up to isomorphism. Therefore from $S_{1} \cong S_{2}$, it follows that $T_{1} \cong T_{2}$. This isomorphism induces an isomorphism of the canonical rings of $T_{1}$ and $T_{2}$. Choose an automorphism $\Phi$ of $\mathbb{P}\left(1^{8}, 2^{8}\right)$ that lifts the isomorphism between Proj $R\left(T_{1}, K_{T_{1}}\right)$ and Proj $R\left(T_{2}, K_{T_{2}}\right)$. Note that $\Phi$ is not unique, as the image by $\Phi^{\sharp}$ of each generator of the underlying polynomial ring is determined only modulo the ideal of $T_{2}$ and therefore, in particular, $\Phi^{\sharp}\left(x_{i a}\right)$ is determined only up to $x_{00}+x_{10}$. In what follows we show that $\Phi_{\mid\left(x_{00}+x_{01}=0\right)}$ belongs to a finite set.

The restriction of the isomorphism $\Phi^{\sharp}$ to the variables of degree 1 yields an automorphism of $\mathbb{P}^{7}$, which we denote by $\tilde{\Phi}$, mapping the canonical image of $T_{1}$ to the canonical image of $T_{2}$. In particular $\tilde{\Phi}$ preserves the hyperplane $\left(x_{00}+x_{01}=0\right)$, which is the linear span of both surfaces, and $W \subset \mathbb{P}^{7}$, given by (2.1), which is their quadric hull. Recall that, as in Definition 2.2, for every $(a, b, c, d) \in \mathcal{L}$, $H_{a b c d}$ is the divisor of poles of the rational function $\varphi^{\sharp}\left(y_{a b c d}\right)=\varphi_{a b c d}$ on $W$. Let us consider the 8 planes given by $\Pi_{a b c d}:=H_{a b c d} \cap\left(x_{00}+x_{01}=0\right) \subset W$. 
Then $\tilde{\Phi}^{-1}\left(\Pi_{a b c d}\right)$ is a plane, it is contained in $W$, and it is the intersection of $\left(x_{00}+x_{01}=0\right)$ with the divisor of the poles of $\varphi^{\sharp} \Phi^{\sharp}\left(y_{a b c d}\right)$. Since $\Phi^{\sharp}\left(y_{a b c d}\right) \in$ $\mathrm{S}^{2}\left\langle x_{i a}\right\rangle \oplus\left\langle y_{a b c d}\right\rangle$, we deduce that $\tilde{\Phi}^{-1}$ permutes the 8 planes $\Pi_{a b c d}$. The first consequence is that $\tilde{\Phi}^{-1}$ preserves the linear span of these 8 planes, $\left(x_{00}=x_{10}=\right.$ $0)$. Now, as we are only interested on $\Phi_{\mid\left(x_{00}+x_{01}=0\right)}$, we may modify $\Phi$ so that $\Phi^{\sharp}\left(x_{0 a}\right)=\lambda x_{0 a}$, for $a=0,1$ and for some $\lambda \in \mathbb{C}^{*}$. Then, rescaling $\Phi^{\sharp}$, and thus still without changing $\Phi_{\mid\left(x_{00}+x_{01}=0\right)}$, we may finally assume that $\Phi^{\sharp}\left(x_{0 a}\right)=$ $x_{0 a}$. Another consequence of the fact that $\tilde{\Phi}^{-1}$ permutes the 8 planes $\Pi_{a b c d}$ is that there exists $\tau \in \mathfrak{S}_{3},(a, b, c, d) \in \mathcal{L}$, with $a=0$ and $\lambda_{1}, \lambda_{2}, \lambda_{3} \in \mathbb{C}^{*}$ such that $\Phi^{\sharp}\left(x_{10}\right)=\lambda_{1} x_{\tau(1) b}, \Phi^{\sharp}\left(x_{20}\right)=\lambda_{2} x_{\tau(2) c}$ and $\Phi^{\sharp}\left(x_{30}\right)=\lambda_{3} x_{\tau(3) b}$. Since $\Phi^{\sharp}$ must also preserve (2.1) we deduce that $\Phi^{\sharp}\left(x_{11}\right)=\lambda_{1}^{-1} x_{\tau(1) b^{\prime}}, \Phi^{\sharp}\left(x_{21}\right)=\lambda_{2}^{-1} x_{\tau(2) c^{\prime}}$ and $\Phi^{\sharp}\left(x_{31}\right)=\lambda_{3}^{-1} x_{\tau(3) b^{\prime}}$.

Consider the action of $\mathfrak{S}_{3}$ on $\mathbb{P}\left(1^{8}, 2^{8}\right)$ given, for every $\tau \in \mathfrak{S}_{3}$ by,

$$
\tau^{\sharp}\left(x_{0 a}\right)=x_{0 a}, \quad \tau^{\sharp}\left(x_{i a}\right)=x_{\tau(i) a}, \quad \tau^{\sharp}\left(y_{a_{0} a_{1} a_{2} a_{3}}\right)=y_{a_{0} a_{\tau^{-1}(1)} a_{\tau^{-1}(2)} a_{\tau^{-1}(3)},},
$$

for all $1 \leq i \leq 3$ and $(a, b, c, d) \in \mathcal{L}$. We note that given $\tau \in \mathfrak{S}_{3}$, we have $\tau \alpha_{i} \tau^{-1}=\alpha_{\tau(i)}$ and $\tau \beta_{i} \tau^{-1}=\beta_{\tau(i)}$, where, recall, $\alpha_{i}$ and $\beta_{i}$ generate $(\mathbb{Z} / 2)^{6}$ and act on $\mathbb{P}\left(1^{8}, 2^{8}\right)$ as given in Table 2.1. These actions generate a finite group $\Lambda$ of automorphisms of $\mathbb{P}\left(1^{8}, 2^{8}\right)$ preserving $V$ and $Y$ which is a semidirect product, $\Lambda \cong(\mathbb{Z} / 2)^{6} \rtimes \mathfrak{S}_{3}$. Accordingly, going back to $\Phi$, there exists $\Psi \in \Lambda$ and constants $\lambda_{i a} \in \mathbb{C}^{*}$ such that

$$
(\Phi \circ \Psi)^{\sharp}\left(x_{i a}\right)=\left(\Psi^{\sharp} \circ \Phi^{\sharp}\right)\left(x_{i a}\right)=\lambda_{i a} x_{i a}
$$

with $\lambda_{00}=\lambda_{01}=1$ and $\lambda_{i 1}=\lambda_{i 0}^{-1}$. Notice that from the cubic relations (2.4) we get $(\Phi \circ \Psi)^{\sharp}\left(y_{a b c d}\right)=\lambda_{1 b^{\prime}} \lambda_{2 c^{\prime}} \lambda_{3 d^{\prime}} y_{a b c d}$. Since for both $T_{1}, T_{2} \in \mathcal{U} \subset \mathcal{N}$ we must have $v_{4} \neq 0$ (for otherwise $T_{1}$ or $T_{2}$ would be too singular), from the equation of the quadric section (2.15), we deduce that the products $\lambda_{1 b^{\prime}} \lambda_{2 c^{\prime}} \lambda_{3 d^{\prime}}$, for $(a, b, c, d) \in \mathcal{L}$ are all equal. This can only happen if $\lambda_{i 1}=\lambda_{i 0}$ for $i \in\{1,2,3\}$. Hence $\lambda_{i a} \in\{ \pm 1\}$, for $i=1,2,3$ and thus there are only finitely many possibilities for $\Phi_{\mid\left(x_{00}+x_{01}\right)=0} \circ \Psi$. Since $\Psi$ belongs to the finite group $\Lambda$, we deduce that there are, as well, only finitely many possibilities for $\Phi_{\mid\left(x_{00}+x_{01}\right)=0}$.

\section{The bicanonical map of $S$}

The main goal of this section is to compare the surfaces we have constructed with the other constructions existing in literature. To reach this goal we study the bicanonical map of $S$, which is interesting in its own right. We show that, as in the Burniat case, the bicanonical map of $S$ is a bidouble cover of a cubic surface in $\mathbb{P}^{3}$ with 3 nodes. This induces a birational description of these surfaces as bidouble covers of the plane. We compute the branch divisors, and then identify the Burniat surfaces. Not surprisingly, the branch divisors corresponding to a general surface in 
our family (cf. Figure 4) correspond exactly to the one used in the recent paper [5] to define the extended tertiary Burniat surfaces.

Consider the action of $(\mathbb{Z} / 2)^{6}$ on $V$ as given in Table 2.1. For a subgroup of $(\mathbb{Z} / 2)^{6}$ to act on $T$ it must preserve the equation $q=0$. An element of $(\mathbb{Z} / 2)^{6}$, written as $\alpha_{1}^{a_{1}} \alpha_{2}^{a_{2}} \alpha_{3}^{a_{3}} \beta_{1}^{b_{1}} \beta_{2}^{b_{2}} \beta_{3}^{b_{3}}$, sends $q=0$ to a scalar multiple of it if and only if the integer $a_{1}+a_{2}+a_{3}+b_{1}+b_{2}+b_{3}$ is even. Let $H$ be the subgroup of $(\mathbb{Z} / 2)^{6}$ given by

$H=\left\{\alpha_{1}^{a_{1}} \alpha_{2}^{a_{2}} \alpha_{3}^{a_{3}} \beta_{1}^{b_{1}} \beta_{2}^{b_{2}} \beta_{3}^{b_{3}} \in(\mathbb{Z} / 2)^{6} \mid a_{1}+a_{2}+a_{3}+b_{1}+b_{2}+b_{3}\right.$ is even $\}$.

The group $G$ defined in (2.10) is obviously a subgroup of $H$. Hence the quotient $\Gamma:=H / G \cong(\mathbb{Z} / 2)^{2}$ acts on $S=T / G$. Denote by $\gamma: S \rightarrow S / \Gamma$ the quotient morphism. In the next proposition we show that $\gamma$ is the bicanonical map of $S$.

Proposition 4.1. Let $T \in \mathcal{N}$ be a surface with at most canonical singularities and such that the action of $G$ on it is fixed-point free. Consider $S=T / G$. Then, the bicanonical map of $S$ is a bidouble cover of the cubic surface $S_{3} \subset \mathbb{P}^{3}$ given by $8 v_{4}^{2}\left(s_{1}-s_{0}\right)\left(s_{2}-s_{0}\right)\left(s_{3}-s_{0}\right)-s_{0}\left(v_{0} s_{0}+v_{1} s_{1}+v_{2} s_{2}+v_{3} s_{3}\right)^{2}=0$.

Proof. The bicanonical system of $S$ is generated by the 4 invariants quadratic forms $s_{0}, s_{1}, s_{2}, s_{3}$. We showed in the proof of Theorem 2.10 that $s_{0}=s_{1}=s_{2}=s_{3}=0$ cuts out the empty set on $T$; therefore $\left|2 K_{S}\right|$ has no fixed part and no base points. Since $S$ is a minimal surface of general type with $p_{g}=0$ and $K^{2} \geq 2$, by [34], the bicanonical system is not composed with a pencil. Hence the image of $\varphi_{2 K_{S}}$ is a surface. To find its equation, we square both sides of (2.17):

$$
v_{4}^{2}\left(x_{10}+x_{11}\right)^{2}\left(x_{20}+x_{21}\right)^{2}\left(x_{30}+x_{31}\right)^{2}=x_{00}^{2} l^{2}
$$

and use $\left(x_{i 0}+x_{i 1}\right)^{2}=2\left(s_{i}+t_{i}\right)=2\left(s_{i}-s_{0}\right)$, for $i=1,2,3$, and $s_{0}=x_{00}^{2}, c f .(2.13)$, (2.14). Substituting, we get $8 v_{4}^{2}\left(s_{1}-s_{0}\right)\left(s_{2}-s_{0}\right)\left(s_{3}-s_{0}\right)-s_{0} l^{2}=0$. For a general choice of $v_{0}, v_{1}, v_{2}, v_{3}, v_{4}$ this cubic is irreducible, hence the cubic surface $S_{3} \subset \mathbb{P}^{3}$ it defines coincides with $\varphi_{2 K_{S}}(S) . S$ has no $(-2)$-curves, as by construction $K_{S}$ is ample, thus $\varphi_{2 K_{S}}$ is a finite morphism of degree 4 . Since $s_{0}, s_{1}, s_{2}, s_{3}$ are invariant for the action of $H$ on $T, \varphi_{2 K_{S}}$ factors through $\gamma$, which is also a finite morphism of degree 4. Hence, since $S_{3}$ is normal ( $c f$. Remark 4.2), $S / \Gamma \cong S_{3}$ and, up to isomorphism, $\varphi_{2 K_{S}}=\gamma$.

Remark 4.2. For general $v_{0}, \ldots, v_{4}$, the cubic $S_{3} \subset \mathbb{P}^{3}$ has 3 ordinary double points:

$$
\begin{aligned}
& n_{1}=\left(s_{2}-s_{0}=s_{3}-s_{0}=v_{0} s_{0}+v_{1} s_{1}+v_{2} s_{2}+v_{3} s_{3}=0\right), \\
& n_{2}=\left(s_{1}-s_{0}=s_{3}-s_{0}=v_{0} s_{0}+v_{1} s_{1}+v_{2} s_{2}+v_{3} s_{3}=0\right), \\
& n_{3}=\left(s_{1}-s_{0}=s_{2}-s_{0}=v_{0} s_{0}+v_{1} s_{1}+v_{2} s_{2}+v_{3} s_{3}=0\right) ;
\end{aligned}
$$

and these are the only singularities of $S_{3}$. 
Let us denote by $\theta_{i} \in \Gamma=H / G$ the class of $\alpha_{i} \beta_{i}$, i.e.,

$$
\theta_{i}=\left[\alpha_{i} \beta_{i}\right]=\left\{\alpha_{i} \beta_{i} g \mid g \in G\right\} .
$$

By Proposition 4.1, $\varphi_{2 K_{S}}$ is the quotient by the action of $\Gamma=\left\{1, \theta_{1}, \theta_{2}, \theta_{3}\right\}$. To study this map, by the general theory of the bidouble covers (see [15]), we study its branch locus. A ramification point of $\varphi_{2 K_{S}}$ is the image of a point $\mathbf{x} \in S$ fixed by some $\theta_{i}$, i.e., for which $I_{\mathbf{x}} \neq\{1\}$, where $I_{\mathbf{x}}=\{g \in \Gamma \mid g \mathbf{x}=\mathbf{x}\}$ is the inertia group of $\mathbf{x}$. When $S$ is smooth, there are 3 possibilities for $I_{\mathbf{x}}$ :

(a) $I_{\mathbf{x}}=\left\langle\theta_{i}\right\rangle$ and $\mathbf{x}$ is an isolated fixed point of $\theta_{i}$. Then, in suitable local coordinates, $\theta_{i}$ acts by $\left(z_{1}, z_{2}\right) \mapsto\left(-z_{1},-z_{2}\right)$ and $\varphi_{2 K_{S}}(\mathbf{x})$ is a node.

(b) $I_{\mathbf{x}}=\left\langle\theta_{i}\right\rangle$ and $\mathbf{x}$ is not an isolated fixed point of $\theta_{i}$. Then, in local coordinates, $\theta_{i}$ acts by $\left(z_{1}, z_{2}\right) \mapsto\left(-z_{1}, z_{2}\right)$ and the locus of all such points is a smooth curve $R_{i} \subset S$.

(c) $I_{\mathbf{x}}=\Gamma$. Then $\mathbf{x}$ belongs to exactly two $R_{i}$ 's, intersecting transversally in $\mathbf{x}$.

Let $D_{i}:=\varphi_{2 K_{S}}\left(R_{i}\right)$ and denote by $\Delta_{i}$ the image of the set of isolated fixed points of $\theta_{i}$ the inertia group of which is not the whole of $\Gamma-$ as in type (a), above. Then each $\Delta_{i}$ is a set of nodes of $S_{3}$. The bidouble cover is determined by $D_{1}, D_{2}, D_{3}$ and $\Delta_{1}, \Delta_{2}, \Delta_{3}$.

To describe $D_{i}$ we introduce some notation. The intersection of $S_{3}$ with the plane $s_{0}+s_{i}=0$ splits as the union of a line with a conic. Denote these by $L_{i}$ and $C_{i}$, respectively. In other words, set

$$
\begin{gathered}
L_{i}=\left(s_{0}=s_{i}=0\right) \text { and } \\
C_{i}=\left(s_{0}+s_{i}=16 v_{4}^{2}\left(s_{i+1}-s_{0}\right)\left(s_{i+2}-s_{0}\right)+l^{2}=0\right),
\end{gathered}
$$

taking the indices in $\{1,2,3\}$, modulo 3 .

Proposition 4.3. Let $D_{i}, \Delta_{i}$, for $i=1,2,3$, be the branch loci of the map $\varphi_{2 K_{S}}: S \rightarrow$ $S_{3} \subset \mathbb{P}^{3}$. Then $\Delta_{i}=\left\{n_{i}\right\}$ and $D_{i}=C_{i+1}+L_{i-1}$, taking indices in $\{1,2,3\}$, modulo 3.

Proof. By cyclic symmetry, it is enough to compute $\Delta_{1}$ and $D_{1}$. On the other hand, the fixed points of $\theta_{1}$ are the images on $S$ of the points of $T$ fixed by an element of $\left[\alpha_{1} \beta_{1}\right]$. Recall that the elements of $\theta_{1}=\left[\alpha_{1} \beta_{1}\right]$ are $\alpha_{1} \beta_{1}, \beta_{1} \beta_{2}, \alpha_{1} \alpha_{2} \beta_{1} \beta_{3}, \alpha_{1} \alpha_{3}$, $\alpha_{2} \beta_{1} \beta_{2} \beta_{3}, \alpha_{3} \beta_{2}, \alpha_{1} \alpha_{2} \alpha_{3} \beta_{3}$ and $\alpha_{2} \alpha_{3} \beta_{2} \beta_{3}$.

$$
\begin{gathered}
\operatorname{Fix}_{(+,+)}\left(\alpha_{2} \beta_{1} \beta_{2} \beta_{3}\right) \text { and } \operatorname{Fix}_{(-,+)}\left(\alpha_{2} \beta_{1} \beta_{2} \beta_{3}\right) \text { are given by } \\
\left(x_{00}-x_{01}=x_{10}=x_{11}=x_{20}+x_{21}=x_{30}=x_{31}=y_{a b c d}+y_{a^{\prime} b c^{\prime} d}=0, \forall_{a b c d \in \mathcal{L}}\right), \\
\left(x_{00}+x_{01}=x_{20}-x_{21}=y_{a b c d}+y_{a^{\prime} b c^{\prime} d}=0, \forall_{a b c d \in \mathcal{L}}\right),
\end{gathered}
$$

respectively. We have $\operatorname{Fix}_{(+,+)}\left(\alpha_{2} \beta_{1} \beta_{2} \beta_{3}\right) \cap T=\emptyset$. This can be seen either directly on $T$ or by noticing that its image in $S_{3}$ must have $s_{0}=s_{1}=s_{3}=0$ and, by (2.1), 
$s_{2}=0$. In $\operatorname{Fix}_{(-,+)}\left(\alpha_{2} \beta_{1} \beta_{2} \beta_{3}\right) \cap T$ we have $x_{00}=-x_{01}$ and $x_{20}=x_{21}$, which by (2.1) imply that $s_{0}+s_{2}=0$. We deduce that the image of $\operatorname{Fix}_{(-,+)}\left(\alpha_{2} \beta_{1} \beta_{2} \beta_{3}\right) \cap T$ in $S_{3}$ is contained in $L_{2} \cup C_{2}$. Suppose that $s_{0}=s_{2}=0$. Then

$$
x_{00}=x_{01}=x_{20}=x_{21}=x_{10} x_{11}=x_{30} x_{31}=0 .
$$

Assume that $x_{10}=x_{30}=0$. Then using (2.5), we get $y_{a b c d}^{2}=-y_{a b c d} y_{a^{\prime} b c^{\prime} d}=0$, for all $(a, b, c, d) \in \mathcal{L} \backslash\{(0,0,0,0),(1,0,1,0)\}$. Hence we are left with the 2 equations:

$$
-y_{0000}^{2}=x_{11}^{2} x_{31}^{2}=4 s_{1} s_{3} \text { and } 2 v_{4} y_{0000}+l=0,
$$

given by (2.5) and $q=0$. Eliminating $y_{0000}$, we get the equation of $C_{2}$ with $s_{0}=s_{2}=0$. This is independent of the choices we made. We deduce that the image of $\operatorname{Fix}_{(-,+)}\left(\alpha_{2} \beta_{1} \beta_{2} \beta_{3}\right) \cap T$ is contained in $C_{2}$. To see that the image of this locus coincides with $C_{2}$ it suffices to check that it is 1-dimensional. The equations $x_{00}+x_{01}=0$ and $x_{20}-x_{21}=0$ define in $W$ a 2-dimensional subscheme (in fact, $x_{00}+x_{01}$ is an equation of $W$ ). It is clear that this subscheme is not contained in the exceptional locus of $\varphi: W \rightarrow V$. Denote by $Z$ its strict transform in $V$. Assume $x_{00}, x_{01} \neq 0$. Then

$$
y_{a b c d} x_{0 a}=x_{1 b^{\prime}} x_{2 c^{\prime}} x_{3 d^{\prime}}=x_{1 b^{\prime}} x_{2 c} x_{3 d^{\prime}}=-y_{a^{\prime} b c^{\prime} d} x_{0 a^{\prime}} \Longrightarrow y_{a b c d}=-y_{a^{\prime} b c^{\prime} d} .
$$

Hence on the open set $x_{00} \neq 0$ of $Z \subset V$, the equations $y_{a b c d}+y_{a^{\prime} b c^{\prime} d}=0$ are redundant. Hence $\operatorname{dim} Z=2$. Since we obtain $T$ from $V$ by taking a hypersurface section $(q=0)$ we deduce that $\operatorname{Fix}_{(-,+)}\left(\alpha_{2} \beta_{1} \beta_{2} \beta_{3}\right) \cap T$ is 1-dimensional. We conclude that the fixed points of $\alpha_{2} \beta_{1} \beta_{2} \beta_{3}$ do not contribute to $\Delta_{1}$ and that their contribution to $D_{1}$ is $C_{2}$.

$$
\begin{aligned}
& \operatorname{Fix}_{(+,+)}\left(\beta_{1} \beta_{2}\right) \text { and } \operatorname{Fix}_{(-,+)}\left(\beta_{1} \beta_{2}\right) \text { are given by: } \\
& \left(x_{10}=x_{11}=x_{20}=x_{21}=0\right) \quad \text { and } \quad\left(x_{00}=x_{01}=x_{30}=x_{31}=0\right),
\end{aligned}
$$

respectively. The image of $\operatorname{Fix}_{(-,+)}\left(\beta_{1} \beta_{2}\right) \cap T$ equals $L_{3}=\left(s_{0}=s_{3}=0\right)$ : it is clearly contained in $L_{3}$ and the equality follows since $\operatorname{Fix}_{(-,+)}\left(\beta_{1} \beta_{2}\right) \supset \mathcal{S}_{11}^{12}$ and $\mathcal{S}_{11}^{12} \cap T$ is positive dimensional. Hence $L_{3} \subset D_{1}$. Notice that by symmetry of the indices we have just shown that $L_{1} \subset D_{2}$ and $L_{2} \subset D_{3}$. For the image of $\operatorname{Fix}_{(+,+)}\left(\beta_{1} \beta_{2}\right) \cap T$, in $S_{3}$ we get $s_{1}=s_{2}=0$ and then $s_{0}=0$, which means that $\operatorname{Fix}_{(+,+)}\left(\beta_{1} \beta_{2}\right) \cap T$ consists of the preimages of the point $L_{1} \cap L_{2}$; these are points in $R_{2} \cap R_{3}$ - type (c) above. We conclude that the fixed points of $\beta_{1} \beta_{2}$ do not contribute to $\Delta_{1}$ and that their contribution to $D_{1}$ is $L_{3}$.

$\operatorname{Fix}_{(+,+)}\left(\alpha_{2} \alpha_{3} \beta_{2} \beta_{3}\right)$ and $\operatorname{Fix}_{(-,+)}\left(\alpha_{2} \alpha_{3} \beta_{2} \beta_{3}\right)$ are given by:

$$
\begin{gathered}
\left(x_{20}+x_{21}=x_{30}+x_{31}=y_{a b c d}-y_{a b c^{\prime} d^{\prime}}=0, \forall_{a b c d \in \mathcal{L}}\right), \\
\left(x_{00}=x_{01}=x_{10}=x_{11}=x_{20}-x_{21}=x_{30}-x_{31}=y_{a b c d}-y_{a b c^{\prime} d^{\prime}}=0, \forall_{a b c d \in \mathcal{L}}\right),
\end{gathered}
$$

respectively. The locus $\operatorname{Fix}_{(-,+)}\left(\alpha_{2} \alpha_{3} \beta_{2} \beta_{3}\right) \cap T$ is clearly empty. For the image of the locus $\operatorname{Fix}_{(+,+)}\left(\alpha_{2} \alpha_{3} \beta_{2} \beta_{3}\right) \cap T$ we get $s_{0}-s_{2}=0$ and $s_{0}-s_{3}=0$ and 
then from the equation of $S_{3}, s_{0} l^{2}=0$. If $s_{0}=0$ then $s_{2}=s_{3}=0$ and then in $\operatorname{Fix}_{(+,+)}\left(\alpha_{2} \alpha_{3} \beta_{2} \beta_{3}\right)$ we get $x_{00}=x_{01}=x_{20}=x_{21}=x_{30}=x_{31}=x_{10} x_{11}=0$. From this we deduce that all $y_{a b c d}$ are zero, which together with $q=0$ forces all variables to be zero. Hence $s_{0} \neq 0$ and we must have $l=0$. To show that the image of $\operatorname{Fix}_{(+,+)}\left(\alpha_{2} \alpha_{3} \beta_{2} \beta_{3}\right) \cap T$ coincides with $n_{1}$, as in (4.2), it suffices to show that this locus is nonempty. The equations $x_{20}+x_{21}=x_{30}+x_{31}=0$ define in $W$ a subscheme of dimension 1 which is not contained in the exceptional locus of $\varphi_{\mid W}: W \rightarrow V$, hence in $V$ they define a positive-dimensional subscheme $Z^{\prime} \subset V$. If $x_{00}, x_{01} \neq 0$ then,

$$
y_{a b c d} x_{0 a}=x_{1 b^{\prime}} x_{2 c^{\prime}} x_{3 d^{\prime}}=x_{1 b^{\prime}} x_{2 c} x_{3 d}=y_{a b c^{\prime} d^{\prime}} x_{0 a} \Longrightarrow y_{a b c d}=y_{a b c^{\prime} d^{\prime}},
$$

which means that in the corresponding (nonempty) open set of $Z^{\prime}$ the equations $y_{a b c d}=y_{a b c^{\prime} d^{\prime}}$ of $\operatorname{Fix}_{(+,+)}\left(\alpha_{2} \alpha_{3} \beta_{2} \beta_{3}\right)$ are redundant. Hence $\operatorname{Fix}_{(+,+)}\left(\alpha_{2} \alpha_{3} \beta_{2} \beta_{3}\right) \cap$ $V$ is positive-dimensional. Since $T$ is obtained from $V$ by taking a hypersurface section, we deduce that $\operatorname{Fix}_{(+,+)}\left(\alpha_{2} \alpha_{3} \beta_{2} \beta_{3}\right) \cap T$ is nonempty. We conclude that the fixed points of $\alpha_{2} \alpha_{3} \beta_{2} \beta_{3}$ do not contribute to $D_{1}$ and that their contribution to $\Delta_{1}$ is $\left\{n_{1}\right\}$.

The arguments we have used so far can be used to show that the 5 remaining elements of $\left[\alpha_{1} \beta_{1}\right]$ contribute neither to $\Delta_{1}$ nor to $D_{1}$.

The cubic surface with 3 nodes $S_{3}$ contains exactly 12 lines, as represented in Figure 4.1 (courtesy of [19]).

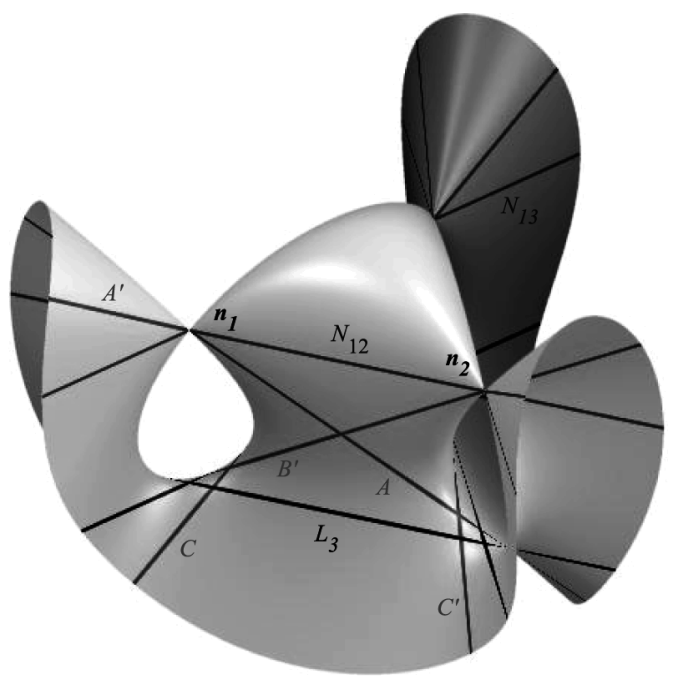

Figure 4.1. Lines on $S_{3}$.

The plane $\left(s_{0}=0\right)$ cut the lines $L_{1}, L_{2}, L_{3}$, forming the bottom triangle in the picture. The plane through the nodes cut the lines

$$
N_{i j}=\left(s_{0}-s_{k}=l=0\right),
$$


where $\{i, j, k\}=\{1,2,3\}$, forming the top triangle. Each $L_{i}$ intersects exactly one of the $N_{i j}: L_{1}$ intersects $N_{23}, L_{2}$ intersects $N_{13}$ and $L_{3}$ intersects $N_{12}$. Consider the plane $\mathcal{P}_{1}$ through $L_{1}$ and $n_{1}$, given by

$$
\left(v_{0}+v_{2}+v_{3}\right) s_{0}+v_{1} s_{1}=0,
$$

and the analogous planes $\mathcal{P}_{2}$ through $L_{2}$ and $n_{2}$ and $\mathcal{P}_{3}$ through $L_{3}$ and $n_{3} . S_{3} \cap \mathcal{P}_{i}$ splits as union of 3 lines: $S_{3} \cap \mathcal{P}_{i}=L_{1} \cup A \cup A^{\prime}, S_{3} \cap \mathcal{P}_{2}=L_{2} \cup B \cup B^{\prime}$ and $S_{3} \cap \mathcal{P}_{3}=L_{3} \cup C \cup C^{\prime}$. We have labeled these last 6 lines as in Figure 4.1, so that $A, B, C$ are pairwise disjoint.

Let $\zeta: \Sigma \rightarrow S_{3}$ be the the blow-up of the 3 nodes, and let $E_{i}$ denote the exceptional divisor of $n_{i}$. With abuse of notation, let us denote by $A, A^{\prime}, B, B^{\prime}, C$, $C^{\prime}, L_{i}, N_{i j}$ the strict transforms in $\Sigma$ of the namesake lines. Similarly we do not change the notation for the strict transforms in $\Sigma$ of $C_{i} \subset S_{3}$. Denote by $H_{\Sigma}$ be the pull-back of an hyperplane section. Since $K_{\Sigma}=-H_{\Sigma}$, the strict transform of every line in $S_{3}$ is a $(-1)$-curve. The curves $N_{12}, N_{13}, N_{23}, A^{\prime}, B^{\prime}, C^{\prime}$ are pairwise disjoint rational curves with self-intersection -1 ; by Castelnuovo's criterion we can contract them to a smooth rational surface with $K^{2}=9$. Therefore, the contraction of these curves yields a morphism $\xi: \Sigma \rightarrow \mathbb{P}^{2}$. Again, with abuse of notation, we shall continue using the same notation for a curve in $S_{3}$, its strict transform in $\Sigma$, and, when it is not contracted to a point, its image in $\mathbb{P}^{2}$. Let us denote by $r_{12}, r_{13}, r_{23}$ and by $\mathbf{x}_{1}, \mathbf{x}_{2}, \mathbf{x}_{3}$, the points of $\mathbb{P}^{2}$ to which $\xi$ contracts $N_{12}, N_{13}, N_{23}$ and $A^{\prime}, B^{\prime}, C^{\prime}$, respectively. In $\mathbb{P}^{2}$ we get the configuration of curves of Figure 4.2.

We leave to the reader the straightforward check that $L_{1}, L_{2}, L_{3}, E_{1}, E_{2}, E_{3}$, $A, B, C$ in $\mathbb{P}^{2}$ are in the configuration of Figure 4.2. As for $C_{2}$, using the equations of $C_{2}$ and $N_{12}$, (4.4) and (4.6), we see that it meets $N_{12}$ in $S_{3}$. Similarly $C_{2}$ meets $N_{23}$. Hence in the plane $C_{2}$ contains the points $r_{12}, r_{23}$. To see that $C_{2}$ contains the points $\mathbf{x}_{1}$ and $\mathbf{x}_{3}$ it is enough to show that, in $S_{3}, C_{2}$ meets the lines $A, A^{\prime}$ and the lines $C, C^{\prime}$. Indeed, as $C_{2}=H_{S_{3}}-L_{2}$ and $A+A^{\prime}=H_{S_{3}}-L_{1}$ in $S_{3}$, we have $C_{2}\left(A+A^{\prime}\right)=\left(H_{S_{3}}-L_{2}\right)\left(H_{S_{3}}-L_{1}\right)=H_{S_{3}}^{2}-H_{S_{3}} L_{1}-H_{S_{3}} L_{2}+L_{1} L_{2}=2$. Likewise one shows that $C_{2}\left(C+C^{\prime}\right)=2$. Additionally, $C_{2}\left(B+B^{\prime}\right)=0$, hence $C_{2}$ does not contain $\mathbf{x}_{2}$. The conics $C_{1}$ and $C_{3}$ have similar properties, obtained by cyclic permutation of the indices $\{1,2,3\}$.

Remark 4.4. Consider the following commutative diagram:

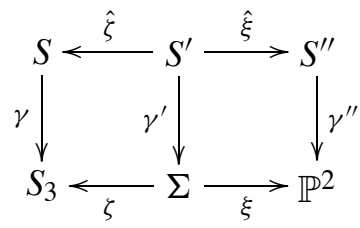

where $S^{\prime}, S^{\prime \prime}, \gamma^{\prime}, \gamma^{\prime \prime}, \hat{\zeta}, \hat{\xi}$ are constructed as fiber products to make both squares cartesian. Notice that $\hat{\xi}$ is the contraction of the preimages of $N_{12}, N_{13}, N_{23}, A^{\prime}$, 




Figure 4.2. The branch divisors of $\gamma^{\prime \prime}: S^{\prime \prime} \rightarrow \mathbb{P}^{2}$.

$B^{\prime}, C^{\prime}$. All horizontal maps in (4.8) are birational morphisms and all vertical maps are bidouble covers. Consider each of the non-trivial involutions of $\Gamma, \theta_{i}$, and denote by $D_{i}^{\prime}$ and $D_{i}^{\prime \prime}$ the images via $\gamma^{\prime}$ and $\gamma^{\prime \prime}$ of the fix locus of $\theta_{i}$. According to Proposition 4.3, $\theta_{i}$ fixes each of the 2 pre-images of the node $n_{i}$. Since these are smooth isolated fixed points for $\theta_{i}, \theta_{i}$ fixes each point in the exceptional divisor of their blow up and accordingly $E_{i}$ is in the branch divisor of $\gamma^{\prime \prime}$ associated with $\theta_{i}$. In conclusion, we have

$$
D_{1}^{\prime \prime}=E_{1}+C_{2}+L_{3} \quad D_{2}^{\prime \prime}=E_{2}+C_{3}+L_{1} \quad D_{3}^{\prime \prime}=E_{3}+C_{1}+L_{2} .
$$

In the Figure 4.2 we have depicted the divisor $D_{1}^{\prime \prime}$ by dashed lines and by dotted lines the divisor $D_{2}^{\prime \prime}$. Note that $S^{\prime \prime}$ is singular and $\hat{\xi}$ is a resolution of its singularities.

Theorem 4.5. A general surface in the 1-dimensional linear subsystem

$$
\mathcal{B}=\left\{T=V \cap(q=0) \mid\left(v_{0}, v_{1}, v_{2}, v_{3}, v_{4}\right)=\left(-v, v, v, v, v_{4}\right), \text { for }\left(v, v_{4}\right) \in \mathbb{P}^{1}\right\} \subset \mathcal{N}
$$

is a surface with 24 isolated rational double points as only singularities and $G$ acts freely on it. The quotient $T / G$ is the canonical model of a tertiary Burniat surface and, conversely, every tertiary Burniat surface arises in this way.

Proof. Analyzing the base locus of $\mathcal{B}$, like in the proof of Theorem 2.10, we get $\operatorname{Sing}(T) \subset\left(l=\sum_{a b c d \in \mathcal{L}}(-1)^{a} y_{a b c d}=0\right)$, where $l=v\left(-s_{0}+s_{1}+s_{2}+s_{3}\right)$. Let $T_{1}, T_{2} \in \mathcal{B}$ be given in $V$ by $s_{0}-s_{1}-s_{2}-s_{3}=0$ and $\sum_{a b c d \in \mathcal{L}}(-1)^{a} y_{a b c d}=0$. 
Fix coordinates $x_{1}, x_{2}, x_{3}$ on $\mathbb{C}^{3}$. Consider the open set $\Omega_{00}=V \backslash\left\{x_{00}=0\right\}$ and the map $\xi_{1}: \Omega_{00} \rightarrow \mathbb{C}^{3}$ given by

$$
\xi_{1}(\mathbf{x}, \mathbf{y})=\left(\frac{x_{10}}{x_{00}}, \frac{x_{20}}{x_{00}}, \frac{x_{30}}{x_{00}}\right) .
$$

Since $x_{00} x_{01} \neq 0$ and (2.1) hold, $\xi_{1}\left(\Omega_{00}\right)$ is contained in $\mathbb{C}^{3} \backslash\left\{x_{1} x_{2} x_{3}=0\right\}$ and the $\operatorname{map} \xi_{2}: \mathbb{C}^{3} \backslash\left\{x_{1} x_{2} x_{3}=0\right\} \rightarrow \Omega_{00}$ given by

$$
\xi_{2}\left(x_{1}, x_{2}, x_{3}\right)=\left(1,-1, x_{1},-1 / x_{1}, x_{2},-1 / x_{2}, x_{3},-1 / x_{3}, \ldots\right),
$$

is the inverse of $\xi_{1}$. Let $\mathcal{B}_{\Omega_{00}}$ denote the pencil $\left\{T \cap \Omega_{00}: T \in \mathcal{B}\right\}$ on $\Omega_{00}$. The pencil $\xi_{2}^{*} \mathcal{B}_{\Omega_{00}}$ is spanned by $\xi_{1}\left(T_{1} \cap \Omega_{00}\right)$ and $\xi_{1}\left(T_{2} \cap \Omega_{00}\right)$, whose equations are:

$$
\begin{gathered}
F_{1}:=1-\frac{1}{2} \sum_{i>0}\left(x_{i}^{2}+1 / x_{i}^{2}\right)=0, \\
F_{2}:=\left(x_{1}-1 / x_{1}\right)\left(x_{2}-1 / x_{2}\right)\left(x_{3}-1 / x_{3}\right)=0,
\end{gathered}
$$

respectively. We show next that a general member of $\xi_{2}^{*} \mathcal{B}_{\Omega_{00}}$ is smooth outside a (fixed) set of 24 rational double points. Since $\frac{\partial F_{1}}{\partial x_{i}}=1 / x_{i}^{3}-x_{i}$, the singularities of $\xi_{1}\left(T_{1} \cap \Omega_{00}\right)$ lie in the set defined by $x_{1}^{4}=x_{2}^{4}=x_{3}^{4}=1$. These equations define 64 points. However only the 24 points of the set

$$
\mathfrak{D}=\{( \pm \epsilon, \pm 1, \pm 1),( \pm 1, \pm \epsilon, \pm 1),( \pm 1, \pm 1, \pm \epsilon)\},
$$

where $\epsilon$ is a square root of -1 , actually belong to $\xi_{1}\left(T_{1} \cap \Omega_{00}\right)$. As $\frac{\partial^{2} F_{1}}{\partial x_{i} \partial x_{j}}=0$, for $i \neq j$, and $\frac{\partial^{2} F_{1}}{\partial x_{i}^{2}}=-3 / x_{i}^{4}-1$, we see that the determinant of the Hessian matrix is nonzero at the points of $\mathfrak{D}$, showing that they are indeed ordinary double points of $\xi_{1}\left(T_{1} \cap \Omega_{00}\right)$. For every point of $\mathfrak{D}$, two factors of $F_{2}$ vanish, thus it is clear that $\xi_{1}\left(T_{2} \cap \Omega_{00}\right)$ is also singular at the points of $\mathfrak{D}$. This shows that a general member of $\xi_{2}^{*} \mathcal{B}_{\Omega_{00}}$ has a rational double point at each point of $\mathfrak{D}$. Since $\xi_{1}\left(T_{1} \cap \Omega_{00}\right)$ is smooth away from $\mathfrak{D}$ it follows that a general member of $\xi_{2}^{*} \mathcal{B}_{\Omega_{00}}$ is also smooth away from $\mathfrak{D}$.

We proceed to show that a general $T \in \mathcal{B}$ is smooth along $T \cap\left(x_{00}=0\right)=T \backslash \Omega_{00}$. If $x_{00}=0$, then $x_{01}=-x_{00}=0$ and, from (2.1), $x_{10} x_{11}=x_{20} x_{21}=x_{30} x_{31}=0$. Let $\Omega_{a b}^{i j}$ denote the open set of $V$ given by $x_{i a} x_{j b} \neq 0$. Since a general $T \in \mathcal{B}$ has $\operatorname{Sing}(T) \subset(l=0)$, if all the variables $x_{00}, x_{01}, \ldots, x_{30}, x_{31}$ but one vanish at a point of $\operatorname{Sing}(T) \cap\left(x_{00}=0\right)$, then from $l=0$ we deduce the remaining one must vanish also. From this, using (2.5) and the fact that $\operatorname{Sing}(T)$ is contained in $T_{2}=\left(\sum_{a b c d \in \mathcal{L}}(-1)^{a} y_{a b c d}=0\right)$, we deduce that all variables must vanish, which is impossible. Hence, for a general $T \in \mathcal{B}$, there exist $i, j, a, b$ with $j>i>0$ such that $\operatorname{Sing}(T) \cap\left(x_{00}=0\right) \subset \Omega_{a b}^{i j}$. Since the role that $x_{10}, x_{11}, x_{20}, x_{21}, x_{30}, x_{31}$ play in the equations of $V$ and $T \in \mathcal{B}$ is symmetric, we 
may reduce to showing that a general member $T \in \mathcal{B}$ is smooth along $T \cap\left(x_{00}=\right.$ $0) \cap \Omega_{11}^{23}$. Similarly to what we did earlier, we consider a map $\zeta_{1}: \Omega_{11}^{23} \rightarrow \mathbb{P}\left(1^{3}, 2\right)$ given by $\zeta_{1}(\mathbf{x}, \mathbf{y})=\left(x_{00}, x_{21}, x_{31}, y_{0000}\right)$. This map has image the (affine) open set defined by $x_{21} x_{31} y_{0000} \neq 0$. This is a consequence of the quartic relation $y_{0000} y_{1100}-x_{21}^{2} x_{31}^{2}$ in (2.5) which holds in $V$. As before, to show that an inverse $\zeta_{2}: \mathbb{P}\left(1^{3}, 2\right) \backslash\left(x_{21} x_{31} y_{0000} \neq 0\right) \rightarrow \Omega_{11}^{23}$ to $\zeta_{1}$ exists, it is enough to express every variable on $\Omega_{11}^{23}$ has a rational function of $x_{00}, x_{21}, x_{31}, y_{0000}$. Using the equations of $V$, i.e., (2.1), (2.4), (2.5) and $x_{00}+x_{01}=0$, on $\Omega_{11}^{23}$ we have:

$$
\begin{aligned}
& x_{01}=-x_{00}, \quad y_{1100}=\frac{x_{21}^{2} x_{31}^{2}}{y_{0000}}, \\
& x_{20}=\frac{x_{00} x_{01}}{x_{21}}=-\frac{x_{00}^{2}}{x_{21}}, \quad x_{30}=\frac{x_{00} x_{01}}{x_{31}}=-\frac{x_{00}^{2}}{x_{31}}, \\
& x_{10}=\frac{x_{01} x_{21} x_{31}}{y_{0000}}=-\frac{x_{00} x_{21} x_{31}}{y_{0000}}, \quad x_{11}=\frac{x_{00} x_{21} x_{31}}{y_{1100}}=\frac{x_{00} y_{0000}}{x_{21} x_{31}} .
\end{aligned}
$$

which are all rational functions of $x_{00}, x_{21}, x_{31}, y_{0000}$. Moreover

$$
y_{a b c 1}=\frac{x_{0 a^{\prime}} x_{1 b^{\prime}} x_{2 c^{\prime}}}{x_{31}} \text { and } y_{a b 1 d}=\frac{x_{0 a^{\prime}} x_{1 b^{\prime}} x_{3 d^{\prime}}}{x_{21}},
$$

which, using (4.14), can be seen to be also rational functions of $x_{00}, x_{21}, x_{31}, y_{0000}$.

Consider $\mathcal{B}_{\Omega_{11}^{23}}$ the pencil $\left\{T \cap \Omega_{11}^{23}: T \in \mathcal{B}\right\}$. Next we show that a general member of $\zeta_{2}^{*} \mathcal{B}_{\Omega_{11}^{23}}$ is smooth along $x_{00}=0$. It suffices to show that $\zeta_{1}\left(T_{1} \cap \Omega_{11}^{23}\right)$ is smooth along $x_{00}=0$. Additionally, since $\zeta_{1}\left(T_{1} \cap \Omega_{11}^{23}\right)$ does not meet the singular point of $\mathbb{P}\left(1^{3}, 2\right)$ we can reduce to showing quasi-smoothness, or, more precisely, non-vanishing of the Jacobian matrix of the polynomial $F_{3}$, obtained from the equation of $\zeta_{1}\left(T_{1} \cap \Omega_{11}^{23}\right)$ by setting $y_{0000}=1$, at the points of $\zeta_{1}\left(T_{1} \cap \Omega_{11}^{23} \cap\right.$ $\left.\left(x_{00}=0\right)\right)$. From $(4.14)$ we deduce $F_{3}=x_{00}^{2} x_{21}^{2} x_{31}^{2}-x_{00}^{2} x_{21}^{4} x_{31}^{4}-x_{00}^{2}-x_{00}^{4} x_{31}^{2}-$ $x_{21}^{4} x_{31}^{2}-x_{00}^{4} x_{21}^{2}-x_{21}^{2} x_{31}^{4}$ using, to ease notation, $x_{00}, x_{21}, x_{31}$ as coordinates for the corresponding affine piece of $\mathbb{P}\left(1^{3}, 2\right)$. Hence, at $x_{00}=0$,

$$
\frac{\partial F_{3}}{\partial x_{21}}=-4 x_{21}^{3} x_{31}^{2}-2 x_{21} x_{31}^{4} \quad \text { and } \quad \frac{\partial F_{3}}{\partial x_{31}}=-2 x_{21}^{4} x_{31}-4 x_{21}^{2} x_{31}^{3}
$$

which have no common zeros for $x_{21} x_{31} \neq 0$.

We have shown that a general member of $\mathcal{B}$ is a smooth away from a set of 24 rational double points given by $\xi_{2}(\mathfrak{D})$ where $\mathfrak{D}$ is the set of points $(4.13)$, in other words, the set of points given in local coordinates by (4.13). Notice that by Remark 2.12, the group $G$ acts freely on a general member of $\mathcal{B}$. To show that $S:=$ $T / G$ for a general $T$ in $\mathcal{B}$ is the canonical model of a Burniat surface we analyze (4.8) for this case in detail. We start by observing that if $\left(v_{0}, v_{1}, v_{2}, v_{3}, v_{4}\right)=$ 
$\left(-v, v, v, v, v_{4}\right)$ then the plane $\mathcal{P}_{1}$ defined in (4.7) is nothing other that the plane $\left(s_{0}+s_{1}=0\right)$ and hence the conic $C_{1}$ splits as $A \cup A^{\prime}$. Likewise, $C_{2}$ splits up as $B \cup B^{\prime}$ and $C_{3}$ splits up as $C \cup C^{\prime}$. Recall that $n_{1} \in A \cap A^{\prime}, n_{2} \in B \cap B^{\prime}$ and $n_{3} \in C \cap C^{\prime}$. Also, the nodes become $n_{1}=(1,-1,1,1), n_{2}=(1,1,-1,1)$ and $n_{3}=(1,1,1,-1)$. Their pre-image in $T$ coincides with the 24 ordinary nodes of $T$. Indeed we see that 8 points of $T$, written in local coordinates of $\Omega_{00} \simeq$ $\mathbb{C}^{3} \backslash\left(x_{1} x_{2} x_{3}=0\right)$ as $( \pm \epsilon, \pm 1, \pm 1)$, map to $n_{1}$; the 8 points $( \pm 1, \pm \epsilon, \pm 1)$ map to $n_{2}$ and the 8 points $( \pm 1, \pm 1, \pm \epsilon)$ map to $n_{3}$. Since $G$ acts freely on $T$, each of these sets of 8 points maps to a single point in $S:=T / G$ which is a node of $S$ and is fixed by every element of $\Gamma$. Denote these 3 nodes of $S$ by $\hat{n}_{1}, \hat{n}_{2}, \hat{n}_{3}$. Using the notation of Proposition 4.3, we claim that

$$
\begin{array}{ll}
\Delta_{1}=\left\{n_{1}, n_{3}\right\}, & D_{1}=B+B^{\prime}+L_{3}, \\
\Delta_{2}=\left\{n_{2}, n_{1}\right\}, & D_{2}=C+C^{\prime}+L_{1}, \\
\Delta_{3}=\left\{n_{3}, n_{2}\right\}, & D_{3}=A+A^{\prime}+L_{2} .
\end{array}
$$

We compute $\Delta_{1}$ and $D_{1}$ by analyzing the fixed loci on $T$ of the elements of:

$$
\left[\alpha_{1} \beta_{1}\right]=\left\{\alpha_{1} \beta_{1}, \beta_{1} \beta_{2}, \alpha_{1} \alpha_{2} \beta_{1} \beta_{3}, \alpha_{1} \alpha_{3}, \alpha_{2} \beta_{1} \beta_{2} \beta_{3}, \alpha_{3} \beta_{2}, \alpha_{1} \alpha_{2} \alpha_{3} \beta_{3}, \alpha_{2} \alpha_{3} \beta_{2} \beta_{3}\right\} .
$$

The computation of $\Delta_{2}, D_{2}, \Delta_{3}$ and $D_{3}$ follows by symmetry. Recall from the proof of Proposition 4.3 that $\operatorname{Fix}\left(\beta_{1} \beta_{2}\right) \cap T$ maps to $L_{3}$ on $S_{3}$, Fix $\left(\alpha_{2} \beta_{1} \beta_{2} \beta_{3}\right) \cap T$ maps to $C_{2}$ which is now $B \cup B^{\prime}$ and that $\operatorname{Fix}\left(\alpha_{2} \alpha_{3} \beta_{2} \beta_{3}\right) \cap T$ maps to $n_{1}$. With the assumptions of that proposition, all of the other elements of $\left[\alpha_{1} \beta_{1}\right]$ have empty fixed locus on $T$. In case of $T \in \mathcal{B}$ this is no longer true. Indeed all but $\alpha_{1} \alpha_{2} \alpha_{3} \beta_{3}$ have empty fixed locus on $T$. To see this, recall that Fix $(+,+)\left(\alpha_{1} \alpha_{2} \alpha_{3} \beta_{3}\right)$ and $\operatorname{Fix}_{(-,+)}\left(\alpha_{1} \alpha_{2} \alpha_{3} \beta_{3}\right)$ are given by

$$
\begin{aligned}
& \left(x_{00}-x_{01}=x_{10}-x_{11}=x_{20}-x_{21}=x_{30}+x_{31}=y_{a b c d}+y_{a^{\prime} b c^{\prime} d}=0, \forall_{a b c d \in \mathcal{L}}\right), \\
& \left(x_{00}+x_{01}=x_{10}+x_{11}=x_{20}+x_{21}=x_{30}-x_{31}=y_{a b c d}-y_{a b c^{\prime} d^{\prime}}=0, \forall_{a b c d \in \mathcal{L}}\right),
\end{aligned}
$$

respectively. It is easy to see that $\operatorname{Fix}_{(+,+)}\left(\alpha_{1} \alpha_{2} \alpha_{3} \beta_{3}\right) \cap T$ is empty. However the locus $\operatorname{Fix}_{(-,+)}\left(\alpha_{1} \alpha_{2} \alpha_{3} \beta_{3}\right)$ now contains the set of points $\{( \pm 1, \pm 1, \pm \epsilon)\}$, given in local coordinates in $\Omega_{00}$. Since $n_{1}$ and $n_{3}$ are the only isolated fixed points of $\theta_{1}$ (notice that $n_{2} \in B \cap B^{\prime}$ ) we have $\Delta_{1}=\left\{n_{1}, n_{3}\right\}$ and $D_{1}=B+B^{\prime}+L_{3}$.

We claim that the branch loci of $\gamma^{\prime}: S^{\prime} \rightarrow \Sigma$ are:

$$
\begin{aligned}
& D_{1}^{\prime}=E_{3}+B+B^{\prime}+L_{3}, \\
& D_{2}^{\prime}=E_{2}+C+C^{\prime}+L_{1}, \\
& D_{3}^{\prime}=E_{1}+A+A^{\prime}+L_{2} .
\end{aligned}
$$

Again by symmetry it is enough to compute $D_{1}^{\prime}$. To do this we must analyze the action of $\theta_{1}$ on the tangent cone at $\hat{n}_{1}, \hat{n}_{2}$ and $\hat{n}_{3}$, showing that it fixes every tangent direction in the tangent cone at $\hat{n}_{3}$ and that it does not act in this way on the tangent 
cones at the nodes $\hat{n}_{1}$ and $\hat{n}_{2}$. This will mean that the action of $\theta_{1}$ on $S^{\prime}$ will fix pointwise $E_{3}$ and will not fix pointwise $E_{1}$ and $E_{2}$. (Recall that $S^{\prime}$ can be obtained by blowing up $\hat{n}_{1}, \hat{n}_{2}, \hat{n}_{3}$.) To analyze the action of $\theta_{1}$ on each of the nodes we will study the action of the corresponding involutions of $\left[\alpha_{1} \beta_{1}\right]$ on the local model given by $\Omega_{00} \subset V$. As we showed earlier, the involutions in $\left[\alpha_{1} \beta_{1}\right]$ which fix in $T$ points in the pre-image of $\hat{n}_{1}, \hat{n}_{2}, \hat{n}_{3}$ are $\alpha_{2} \alpha_{3} \beta_{2} \beta_{3}, \alpha_{2} \beta_{1} \beta_{2} \beta_{3}$ and $\alpha_{1} \alpha_{2} \alpha_{3} \beta_{3}$. We have seen that $\alpha_{2} \beta_{1} \beta_{2} \beta_{3}$ fixes a positive dimensional locus containing the pre-images of $\hat{n}_{2}$, hence, locally at each pre-image, this involution cannot fix every tangent direction to it and therefore $E_{2}$ is not in $D_{1}^{\prime}$. The involution $\alpha_{2} \alpha_{3} \beta_{2} \beta_{3}$, whose fixed locus on $T$ maps to $\left\{\hat{n}_{1}\right\}$, can be written in the local model $\mathbb{C}^{3} \backslash\left(x_{1} x_{2} x_{3}=0\right) \simeq \Omega_{00}$ as:

$$
\left(x_{1}, x_{2}, x_{3}\right)=\left(\frac{x_{10}}{x_{00}}, \frac{x_{20}}{x_{00}}, \frac{x_{30}}{x_{00}}\right) \mapsto\left(\frac{x_{10}}{x_{00}},-\frac{x_{21}}{x_{00}},-\frac{x_{31}}{x_{00}}\right)=\left(x_{1}, \frac{1}{x_{2}}, \frac{1}{x_{3}}\right) .
$$

(Recall that $x_{00}+x_{01}=0$ and $x_{00} x_{01}=x_{i 0} x_{i 1} \Longrightarrow x_{00} / x_{i 0}=x_{i 1} / x_{01}$.) We see that $( \pm \epsilon, \pm 1, \pm 1)$ are fixed. However we see also that the fixed loci of this involution in the ambient $\mathbb{C}^{3} \backslash\left(x_{1} x_{2} x_{3}=0\right)$ is a set of four lines, going through the 8 points $( \pm \epsilon, \pm 1, \pm 1)$. Hence this involution does not fix all of the tangent directions at any of these points. This implies that $\theta_{1}$ does not fix all of the tangent directions of $\hat{n}_{1}$ and thus $E_{1}$ is also not in $D_{1}^{\prime}$. Finally, writing $\alpha_{1} \alpha_{2} \alpha_{3} \beta_{3}$ in the local model:

$$
\left(x_{1}, x_{2}, x_{3}\right)=\left(\frac{x_{10}}{x_{00}}, \frac{x_{20}}{x_{00}}, \frac{x_{30}}{x_{00}}\right) \mapsto\left(\frac{x_{11}}{x_{01}}, \frac{x_{21}}{x_{01}},-\frac{x_{31}}{x_{01}}\right)=\left(\frac{1}{x_{1}}, \frac{1}{x_{2}},-\frac{1}{x_{3}}\right),
$$

we see that this involution fixes exactly the set of points given in local coordinates by $\{( \pm 1, \pm 1, \pm \epsilon)\}$. This coincides with the pre-image of $\left\{\hat{n_{3}}\right\}$. Moreover, since it fixes only finitely many points, it must fix every tangent direction at each of these points. We conclude that $\theta_{3}$ fixes every tangent direction of $\hat{n}_{3}$ and thus $D_{1}^{\prime}=$ $E_{3}+B+B^{\prime}+L_{3}$.

It is now easy to compute the ramification divisors of $\gamma^{\prime \prime}: S^{\prime \prime} \rightarrow \mathbb{P}^{2}$. Since $S^{\prime \prime}$ can be obtained by contracting the pre-images of $N_{23}, N_{13}, N_{12}, A^{\prime}, B^{\prime}, C^{\prime}$ it is clear that

$$
\begin{aligned}
& D_{1}^{\prime \prime}=E_{3}+B+L_{3}, \\
& D_{2}^{\prime \prime}=E_{2}+C+L_{1}, \\
& D_{3}^{\prime \prime}=E_{1}+A+L_{2} .
\end{aligned}
$$

With the help of Figure 4.2 we see that these are exactly the branch loci for a tertiary Burniat surface.

The space of tertiary Burniat surfaces is parameterized by $\lambda \in \mathbb{C}^{*} \backslash\{1\}$ as follows. In Figure 1.1, we may always choose coordinates $\left(u_{0}, u_{1}, u_{2}\right)$ such that $\mathbf{x}_{1}=(1,0,0), \mathbf{x}_{2}=(0,1,0), \mathbf{x}_{3}=(0,0,1)$ and the further 3 marked points are respectively $(1,1,1),(1,1, \lambda)$ and $(\lambda, 1, \lambda)$. The bicanonical image of the Burniat 
surface is the image of $\mathbb{P}^{2}$ in $\mathbb{P}^{3}$ by the linear system of cubics through the 6 marked points. If we choose, as basis for this system, the cubics

$$
\begin{aligned}
& s_{0}=-\frac{1}{2}\left(u_{0}-\lambda u_{1}\right)\left(u_{1}-u_{2}\right)\left(u_{2}-\lambda u_{0}\right) \\
& s_{1}=(1-\lambda) u_{0}\left(u_{1}-u_{2}\right)\left(\lambda u_{1}-u_{2}\right)-s_{0} \\
& s_{2}=(1-\lambda) u_{1}\left(u_{2}-u_{0}\right)\left(u_{2}-\lambda u_{0}\right)-s_{0} \\
& s_{3}=(1-\lambda) u_{2}\left(u_{0}-u_{1}\right)\left(u_{0}-\lambda u_{1}\right)-s_{0},
\end{aligned}
$$

then one can check that

$$
(\lambda+1)^{2}\left(s_{1}-s_{0}\right)\left(s_{2}-s_{0}\right)\left(s_{3}-s_{0}\right)=-2 \lambda s_{0}\left(s_{3}+s_{2}+s_{1}-s_{0}\right)^{2}
$$

and we easily conclude that the tertiary Burniat surface under consideration is isomorphic to $S=T / G$ with $T \in \mathcal{N}$ given by $-v_{0}=\nu_{1}=v_{2}=\nu_{3}=\sqrt{-\lambda}$ and $v_{4}=4(\lambda+1)$.

\section{References}

[1] S. AltinoK, G. BRown and M. Reid, Fano 3-folds, K3 surfaces and graded rings, In: "Topology and geometry: commemorating SISTAG", Contemp. Math., Vol. 314, Amer. Math. Soc., Providence, RI, 2002, 25-53.

[2] V. AlEXEEV and R. PARDINI, Explicit compactifications of moduli spaces of Campedelli and Burniat surfaces, arxiv: 0901.4431

[3] I. BAUER and F. CATANESE, Burniat surfaces I: fundamental groups and moduli of primary Burniat surfaces, arXiv:0909.3699

[4] I. BAUER and F. CATANESE, Burniat surfaces II: secondary Burniat surfaces form three connected components of the moduli space, Invent. Math. 180 (2010), 559-588.

[5] I. BAUER and F. CATANESE, Burniat surfaces III: deformations of automorphisms and extended Burniat surfaces. arXiv: 1012.3770

[6] I. BAuer, F. CATANeSE and F. GRUnewald, The classification of surfaces with $p_{g}=$ $q=0$ isogenous to a product of curves, Pure Appl. Math. Q. 4 (2008), 547-586.

[7] I. Bauer, F. Catanese, F. GRunewald and R. Pignatelli, Quotients of products of curves, new surfaces with $p_{g}=0$ and their fundamental groups, Amer. J. Math. 134 (2012), 993-1049.

[8] I. BAuer, F. Catanese and R. Pignatelli, Surfaces of general type with geometric genus zero: a survey, In: "Complex and Differential Geometry", Springer Proceedings in Mathematics, Vol. 8, 2011,1-48.

[9] I. BAUER and R. PIGNATELLI, The classification of minimal product-quotient surfaces with $p_{g}=0$, Math. Comp. 81 (2012), 2389-2418.

[10] L. BAYLE, Classification des variétés complexes projectives de dimension trois dont une section hyperplane générale est une surface d'Enriques, J. Reine Angew. Math. 449 (1994), 9-63.

[11] G. BRown, Graded ring database homepage Online searchable database, available from http://grdb.lboro.ac.uk/

[12] G. BRown and K. SuZUKI, Fano 3-folds with divisible anticanonical class, Manuscripta Math. 123 (2007), 37-51.

[13] P. Burniat, Sur les surfaces de genre $P_{12}>1$, Ann. Mat. Pura Appl. (4) 71 (1966), 1-24.

[14] D. I. CARTWRIGHT and T. STEGER, Enumeration of the 50 fake projective planes, C. R. Math. Acad. Sci. Paris 348 (2010), 11-13. 
[15] F. CATANESE, Singular bidouble covers and the construction of interesting algebraic surfaces, In: "Algebraic geometry: Hirzebruch 70" (Warsaw, 1998), Contemp. Math., Vol. 241, Amer. Math. Soc., Providence, RI, 1999, 97-120.

[16] D. EISENBUD, "Commutative Algebra, with a View Toward Algebraic Geometry", Graduate Texts in Mathematics, Vol. 150, Springer-Verlag, 1995.

[17] M. InOUE, Some new surfaces of general type, Tokyo J. Math. 17 (1994), 295-319.

[18] V. S. KULIKOV, Old examples and a new example of surfaces of general type with $p_{g}=0$. (Russian) Izv. Ross. Akad. Nauk Ser. Mat. 68 (2004), 123-170; translation in Izv. Math. 68 (2004), 965-1008.

[19] O. Labs and D. van Straten, "The Cubic Surface Homepage", University of Mainz (2001), www.Cubics.AlgebraicSurface.net

[20] Y. LEE and J. PARK, A simply connected surface of general type with $p_{g}=0$ and $K^{2}=2$, Invent. Math. 170 (2007), 483-505.

[21] M. MENDES LOPES and R. PARDINI, A connected component of the moduli space of surfaces with $p_{g}=0$, Topology 40 (2001), 977-991.

[22] M. Mendes Lopes and R. PARDini, A new family of surfaces with $p_{g}=0$ and $K^{2}=3$, Ann. Sci. École Norm. Sup. (4) 37 (2004), 507-531.

[23] M. Mendes Lopes and R. PARDini, Numerical Campedelli surfaces with fundamental group of order 9, J. Eur. Math. Soc. (JEMS) 10 (2008), 457-476.

[24] M. MENDES LOPES, R. PARDINI and M. REID, Campedelli surfaces with fundamental group of order 8, Geom. Dedicata 139 (2009), 49-55.

[25] Y. MiYAOKA, On numerical Campedelli surfaces, In: "Complex Analysis and Algebraic Geometry", Iwanami Shoten, Tokyo, 1977, 113-118.

[26] J. Neves and S. A. PAPADAKIS, A construction of numerical Campedelli surfaces with torsion $\mathbb{Z} / 6$, Trans. Amer. Math. Soc. 361 (2009), 4999-5021.

[27] J. Neves and S. A. PAPADAKIS, Parallel Kustin-Miller unprojection with an application to Calabi-Yau geometry, Proc. Lond. Math. Soc. (3) 106 (2013), 203-223.

[28] H. PARK, J. PARK and D. SHIN, A simply connected surface of general type with $p_{g}=0$ and $K^{2}=3$, Geom. Topol. 13 (2009), 743-767.

[29] C. A. M. Peters, On certain examples of surfaces with $p_{g}=0$ due to Burniat, Nagoya Math. J. 66 (1977), 109-119.

[30] G. PRASAD and S-K. YEUnG, Fake projective planes. Invent. Math. 168 (2007), 321-370.

[31] G. PRASAD and S-K. YEUNG, Arithmetic fake projective spaces and arithmetic fake Grassmannians, Amer. J. Math. 131 (2009), 379-407.

[32] M. REID, Surfaces with $p_{g}=0, K^{2}=2$,

Preprint available at: http://www.warwick.ac.uk/ masda/surf/.

[33] T. S Ano, On classifications of non-Gorenstein $\mathbb{Q}$-Fano 3-folds of Fano index 1, J. Math. Soc. Japan 47 (1995), 369-380.

[34] G. XIaO, Finitude de l'application bicanonique des surfaces de type général, Bull. Soc. Math. France 113 (1985), 23-51.

CMUC

Department of Mathematics University of Coimbra 3001-454 Coimbra, Portugal neves@mat.uc.pt

Dipartimento di Matematica Università di Trento Via Sommarive 14 loc. Povo I-38050 Trento, Italia Roberto.Pignatelli@unitn.it 